\title{
Age constraints on the timing of iron ore mineralisation in the southeastern Gawler Craton
}

\author{
K. LANE ${ }^{1 *}$, E. JAGODZINSKI ${ }^{2}$, A. REID ${ }^{2}$, M. HAND ${ }^{1}$ AND R. DUTCH ${ }^{2}$
}

${ }^{1}$ Centre for Tectonics, Resources and eXploration (TRaX), School of Earth and Environmental Sciences, The University of Adelaide, Adelaide, SA 5005, Australia ${ }^{2}$ Geological Survey of South Australia, Department of Manufacturing, Innovation, Trade, Resources and Energy, 101 Grenfell Street, Adelaide, SA 5000, Australia

$\left({ }^{*}\right)$ Corresponding author contact: kathleen.lane@adelaide.edu.au

Australian Journal of Earth Sciences (2015) 62, 55-75

http://dx.doi.org/10.1080/08120099.2015.993160

Copies of Supplementary Papers may be obtained from the Geological Society of Australia's website (www.gsa.org.au), the Australian Journal of Earth Scienecs website (www.ajes.com.au) or from the National Library of Australia's Pandora archive (http://nla.gov.au/nla.arc-25194).

\section{SUPPLEMENTARY PAPER}

Table $1 \mathrm{U}-\mathrm{Pb}$ SHRIMP data and Sm-Nd data.

Table $2 \mathrm{Sm}-\mathrm{Nd}$ isotope data. 
Lane et al. Supplementary Papers

Australian Journal of Earth Sciences (2015) 62, 55-75, http://dx.doi.org/10.1080/08120099.2015.993160

Table $1 \mathrm{U}-\mathrm{Pb}$ SHRIMP data.

\begin{tabular}{|c|c|c|c|c|c|c|c|c|c|c|c|}
\hline Spot & ${ }^{232} \mathrm{Th} /{ }^{238} \mathrm{U}$ & $\%{ }^{206} \mathrm{~Pb}_{\mathrm{c}}$ & $\begin{array}{c}{ }^{206} \mathrm{~Pb}^{*} \mathrm{I} \\
{ }^{238} \mathrm{U}\end{array}$ & $\pm \%$ & $\begin{array}{c}{ }^{207} \mathrm{~Pb}^{*} \mathrm{I} \\
{ }^{235} \mathrm{U}\end{array}$ & $\pm \%$ & $\begin{array}{l}{ }^{207} \mathrm{~Pb}^{*} \mathrm{I} \\
{ }^{206} \mathrm{~Pb}^{*}\end{array}$ & $\pm \%$ & $\begin{array}{c}{ }^{207} \mathrm{~Pb} /{ }^{206} \mathrm{~Pb} \\
\mathrm{Age}\end{array}$ & \pm & $\%$ Disc \\
\hline 2058314 & \multicolumn{11}{|c|}{ SHRIMP session 1} \\
\hline \multicolumn{12}{|c|}{ Detrital zircon } \\
\hline 20402-08 & 0.81 & 0.016 & 0.626 & 2.9 & 21.74 & 3.1 & 0.2517 & 1.17 & 3196 & 19 & +2 \\
\hline $20402-35$ & 0.43 & -0.002 & 0.529 & 3.0 & 14.45 & 3.2 & 0.1982 & 0.81 & 2811 & 13 & +3 \\
\hline 20402-39 & 0.91 & 0.026 & 0.492 & 3.2 & 12.20 & 3.2 & 0.1797 & 0.34 & 2650 & 6 & +3 \\
\hline $20402-15$ & 0.60 & 0.168 & 0.499 & 3.0 & 12.30 & 3.1 & 0.1789 & 0.76 & 2643 & 13 & +2 \\
\hline $20402-50$ & 0.95 & 0.100 & 0.496 & 2.9 & 12.22 & 2.9 & 0.1786 & 0.38 & 2640 & 6 & +2 \\
\hline $20402-28$ & 0.29 & 0.061 & 0.494 & 3.0 & 12.08 & 3.2 & 0.1773 & 1.33 & 2628 & 22 & +2 \\
\hline $20402-61$ & 0.07 & 0.060 & 0.487 & 2.9 & 11.76 & 3.2 & 0.1753 & 1.49 & 2609 & 25 & +2 \\
\hline 20402-62 & 0.28 & 0.007 & 0.473 & 3.4 & 11.22 & 5.0 & 0.1721 & 3.66 & 2579 & 61 & +4 \\
\hline 20402-09 & 0.29 & 0.034 & 0.468 & 2.9 & 11.04 & 3.0 & 0.1710 & 0.89 & 2567 & 15 & +4 \\
\hline $20402-54$ & 0.51 & 0.031 & 0.480 & 2.9 & 11.28 & 3.5 & 0.1705 & 1.99 & 2562 & 33 & +2 \\
\hline $20402-58$ & 1.16 & 0.059 & 0.470 & 2.9 & 11.02 & 2.9 & 0.1702 & 0.31 & 2559 & 5 & +4 \\
\hline $20402-46$ & 0.44 & 0.014 & 0.469 & 3.3 & 10.99 & 4.7 & 0.1701 & 3.41 & 2558 & 57 & +4 \\
\hline $20402-21$ & 1.01 & -0.005 & 0.484 & 4.1 & 11.30 & 5.1 & 0.1693 & 2.99 & 2551 & 50 & +0 \\
\hline $20402-40$ & 0.15 & 0.230 & 0.485 & 3.3 & 11.26 & 3.6 & 0.1682 & 1.40 & 2540 & 24 & -0 \\
\hline 20402-59 & 0.69 & 0.127 & 0.471 & 2.9 & 10.92 & 3.0 & 0.1682 & 0.89 & 2540 & 15 & +2 \\
\hline $20402-48$ & 0.27 & 0.064 & 0.459 & 4.7 & 10.61 & 5.0 & 0.1677 & 1.81 & 2535 & 30 & $+\overline{5}$ \\
\hline $20402-51$ & 0.31 & 0.096 & 0.473 & 2.9 & 10.93 & 3.0 & 0.1677 & 0.52 & 2535 & 9 & +2 \\
\hline $20402-55$ & 0.51 & 0.003 & 0.478 & 2.9 & 11.04 & 2.9 & 0.1676 & 0.63 & 2534 & 11 & +1 \\
\hline $20402-67$ & 0.11 & 0.116 & 0.477 & 3.2 & 10.93 & 3.6 & 0.1660 & 1.81 & 2518 & 30 & +0 \\
\hline $20402-56$ & 0.92 & -0.011 & 0.486 & 3.0 & 11.08 & 3.0 & 0.1654 & 0.63 & 2511 & 11 & -2 \\
\hline $20402-41$ & 0.28 & 0.010 & 0.461 & 2.9 & 10.48 & 2.9 & 0.1651 & 0.39 & 2508 & 7 & +3 \\
\hline 20402-18 & 0.12 & -0.012 & 0.456 & 3.8 & 10.36 & 4.0 & 0.1647 & 1.17 & 2505 & 20 & +4 \\
\hline $20402-25$ & 0.12 & 0.014 & 0.458 & 3.3 & 10.37 & 3.9 & 0.1643 & 1.99 & 2500 & 33 & +3 \\
\hline $20402-22$ & 0.13 & 0.031 & 0.457 & 2.9 & 10.34 & 2.9 & 0.1641 & 0.66 & 2499 & 11 & +3 \\
\hline 20402-63 & 0.90 & 0.063 & 0.482 & 3.0 & 10.87 & 3.1 & 0.1638 & 0.94 & 2495 & 16 & -2 \\
\hline $20402-17$ & 0.26 & -0.010 & 0.463 & 2.9 & 10.40 & 2.9 & 0.1628 & 0.40 & 2485 & 7 & +1 \\
\hline $20402-29$ & 0.66 & 0.020 & 0.454 & 2.9 & 10.16 & 2.9 & 0.1623 & 0.47 & 2479 & 8 & +3 \\
\hline $20402-36$ & 0.14 & -0.017 & 0.449 & 3.4 & 10.02 & 3.4 & 0.1620 & 0.47 & 2476 & 8 & +4 \\
\hline $20402-66$ & 0.01 & 0.080 & 0.473 & 2.9 & 10.56 & 3.3 & 0.1619 & 1.59 & 2475 & 27 & -1 \\
\hline 20402-01 & 0.64 & 0.037 & 0.448 & 2.9 & 9.99 & 2.9 & 0.1616 & 0.64 & 2473 & 11 & +4 \\
\hline
\end{tabular}


Lane et al. Supplementary Papers

Australian Journal of Earth Sciences (2015) 62, 55-75, http://dx.doi.org/10.1080/08120099.2015.993160

\begin{tabular}{|c|c|c|c|c|c|c|c|c|c|c|c|}
\hline Spot & ${ }^{232} \mathrm{Th} /{ }^{238} \mathrm{U}$ & $\%{ }^{206} \mathrm{~Pb}_{\mathrm{c}}$ & $\begin{array}{l}{ }^{206} \mathrm{~Pb}^{*} I \\
{ }^{238} \mathrm{U}\end{array}$ & $\pm \%$ & $\begin{array}{l}{ }^{207} \mathrm{~Pb}^{*} \mathrm{I} \\
{ }^{235} \mathrm{U}\end{array}$ & $\pm \%$ & $\begin{array}{l}{ }^{207} \mathrm{~Pb}^{\star} \mathrm{I} \\
{ }^{206} \mathrm{~Pb}^{*}\end{array}$ & $\pm \%$ & $\begin{array}{c}{ }^{207} \mathrm{~Pb} /{ }^{206} \mathrm{~Pb} \\
\mathrm{Age}\end{array}$ & \pm & $\%$ Disc \\
\hline $20402-32$ & 0.13 & 0.012 & 0.458 & 3.2 & 10.12 & 4.1 & 0.1602 & 2.47 & 2458 & 42 & +1 \\
\hline $20402-44$ & 1.05 & 0.317 & 0.457 & 3.1 & 10.06 & 4.2 & 0.1597 & 2.83 & 2453 & 48 & +1 \\
\hline $20402-57$ & 0.45 & 0.040 & 0.445 & 2.9 & 9.75 & 3.1 & 0.1588 & 1.15 & 2443 & 19 & +3 \\
\hline $20402-31$ & 0.13 & 0.027 & 0.456 & 2.9 & 9.95 & 3.5 & 0.1582 & 2.03 & 2437 & 34 & +1 \\
\hline $20402-52$ & 0.12 & 0.051 & 0.452 & 2.9 & 9.87 & 3.0 & 0.1582 & 0.80 & 2436 & 14 & +1 \\
\hline $20402-07$ & 0.23 & -0.007 & 0.446 & 2.9 & 9.66 & 3.0 & 0.1571 & 0.53 & 2424 & 9 & +2 \\
\hline $20402-02$ & 0.22 & 0.321 & 0.437 & 2.9 & 9.40 & 3.0 & 0.1561 & 0.65 & 2414 & 11 & +4 \\
\hline \multicolumn{12}{|c|}{ Discordant detrital zircon/high $\mathrm{Pbc}$} \\
\hline $20402-30$ & 0.32 & 0.004 & 0.462 & 2.9 & 11.52 & 5.7 & 0.1808 & 4.88 & 2661 & 81 & +10 \\
\hline $20402-47$ & 0.01 & 0.085 & 0.474 & 2.8 & 11.81 & 13.6 & 0.1807 & 13 & 2659 & 220 & +7 \\
\hline 20402-04 & 0.65 & -0.010 & 0.469 & 2.9 & 11.23 & 2.9 & 0.1738 & 0.31 & 2595 & 5 & +5 \\
\hline $20402-10$ & 0.20 & 0.192 & 0.444 & 2.9 & 10.59 & 4.5 & 0.1731 & 3.50 & 2588 & 58 & +10 \\
\hline $20402-71$ & 0.44 & 0.071 & 0.574 & 11.6 & 13.63 & 14.9 & 0.1724 & 9.35 & 2581 & 156 & -16 \\
\hline $20402-27$ & 0.16 & 0.012 & 0.462 & 2.9 & 10.91 & 6.1 & 0.1711 & 5.36 & 2568 & 90 & +6 \\
\hline $20402-53$ & 0.55 & 1.108 & 0.422 & 2.9 & 9.91 & 3.0 & 0.1705 & 0.68 & 2563 & 11 & +14 \\
\hline $20402-19$ & 0.45 & 0.027 & 0.459 & 2.9 & 10.70 & 3.4 & 0.1689 & 1.70 & 2547 & 29 & +5 \\
\hline $20402-14$ & 0.08 & 0.023 & 0.446 & 3.0 & 10.37 & 5.6 & 0.1685 & 4.71 & 2543 & 79 & +8 \\
\hline $20402-13$ & 0.17 & 0.000 & 0.438 & 2.9 & 10.13 & 5.0 & 0.1679 & 4.02 & 2537 & 67 & +9 \\
\hline $20402-38$ & 0.29 & 0.163 & 0.447 & 3.0 & 10.30 & 3.1 & 0.1673 & 0.70 & 2530 & 12 & +7 \\
\hline 20402-34 & 0.08 & 0.112 & 0.442 & 2.9 & 10.16 & 4.9 & 0.1668 & 4.00 & 2526 & 67 & +8 \\
\hline 20402-70 & 0.22 & 0.003 & 0.515 & 2.9 & 11.71 & 3.5 & 0.1649 & 1.96 & 2506 & 33 & -8 \\
\hline $20402-45$ & 0.15 & -0.004 & 0.450 & 3.1 & 10.24 & 3.7 & 0.1648 & 2.05 & 2506 & 35 & +5 \\
\hline $20402-60$ & 0.02 & 0.054 & 0.417 & 3.0 & 9.30 & 3.4 & 0.1619 & 1.59 & 2476 & 27 & +11 \\
\hline 20402-20 & 0.69 & 0.282 & 0.431 & 2.9 & 9.59 & 3.0 & 0.1612 & 0.57 & 2469 & 10 & +8 \\
\hline $20402-11$ & 0.18 & 0.055 & 0.440 & 2.9 & 9.75 & 2.9 & 0.1608 & 0.38 & 2464 & 6 & +6 \\
\hline $20402-23$ & 0.16 & 0.026 & 0.423 & 2.9 & 9.36 & 2.9 & 0.1605 & 0.50 & 2461 & 8 & +9 \\
\hline \multicolumn{12}{|c|}{ Metamorphic zircon } \\
\hline $20402-16$ & 0.30 & 0.021 & 0.460 & 3.3 & 10.23 & 3.4 & 0.1612 & 1.15 & 2469 & 19 & +1 \\
\hline $20402-42$ & 0.02 & -0.007 & 0.441 & 3.0 & 9.75 & 3.0 & 0.1602 & 0.59 & 2458 & 10 & +5 \\
\hline $20402-64$ & 0.04 & 0.121 & 0.453 & 2.9 & 9.94 & 2.9 & 0.1590 & 0.44 & 2445 & 7 & +2 \\
\hline 20402-03 & 0.01 & 0.004 & 0.443 & 2.8 & 9.69 & 2.9 & 0.1587 & 0.26 & 2442 & 4 & +4 \\
\hline $20402-43$ & 0.02 & 0.008 & 0.464 & 6.2 & 10.04 & 6.6 & 0.1570 & 2.18 & 2424 & 37 & -2 \\
\hline
\end{tabular}


Lane et al. Supplementary Papers

Australian Journal of Earth Sciences (2015) 62, 55-75, http://dx.doi.org/10.1080/08120099.2015.993160

\begin{tabular}{|c|c|c|c|c|c|c|c|c|c|c|c|}
\hline Spot & ${ }^{232} \mathrm{Th} /{ }^{238} \mathrm{U}$ & ${ }^{\%}{ }^{206} \mathrm{~Pb}_{\mathrm{c}}$ & $\begin{array}{c}{ }^{206} \mathrm{~Pb}^{*} I \\
{ }^{238} \mathrm{U}\end{array}$ & $\pm \%$ & $\begin{array}{c}{ }^{207} \mathrm{~Pb}^{*} \mathrm{I} \\
{ }^{235} \mathrm{U}\end{array}$ & $\pm \%$ & $\begin{array}{l}{ }^{207} \mathrm{~Pb}^{*} \mathrm{I} \\
{ }^{206} \mathrm{~Pb}^{*}\end{array}$ & $\pm \%$ & $\begin{array}{c}{ }^{207} \mathrm{~Pb} /{ }^{206} \mathrm{~Pb} \\
\text { Age }\end{array}$ & \pm & $\%$ Disc \\
\hline \multicolumn{12}{|c|}{ Discordant metamorphic zircon } \\
\hline 20402-05 & 0.07 & 0.053 & 0.428 & 3.2 & 9.40 & 3.2 & 0.1591 & 0.42 & 2446 & 7 & +7 \\
\hline $20402-49$ & 0.02 & 0.290 & 0.488 & 4.5 & 10.66 & 4.7 & 0.1585 & 1.26 & 2439 & 21 & -6 \\
\hline $20402-12$ & 0.09 & -0.016 & 0.428 & 2.9 & 9.25 & 2.9 & 0.1568 & 0.33 & 2421 & 6 & +6 \\
\hline $20402-68$ & 0.09 & 0.006 & 0.510 & 3.1 & 11.00 & 3.4 & 0.1564 & 1.31 & 2418 & 22 & -12 \\
\hline $20402-65$ & 0.29 & 0.185 & 0.488 & 2.9 & 10.34 & 2.9 & 0.1538 & 0.26 & 2388 & 4 & -9 \\
\hline 2058315 & \multicolumn{11}{|c|}{ SHRIMP session 1} \\
\hline \multicolumn{12}{|c|}{ Inherited grains } \\
\hline IR41-62 & 0.74 & -0.006 & 0.522 & 2.9 & 15.00 & 3.2 & 0.2086 & 1.23 & 2894 & 20 & +8 \\
\hline IR41-35 & 0.36 & 0.010 & 0.462 & 2.8 & 11.15 & 2.9 & 0.1748 & 0.58 & 2604 & 10 & +7 \\
\hline IR41-67 & 0.60 & 0.019 & 0.478 & 2.9 & 11.35 & 3.3 & 0.1723 & 1.47 & 2580 & 24 & +3 \\
\hline IR41-25 & 0.33 & 0.208 & 0.439 & 2.9 & 10.33 & 2.9 & 0.1709 & 0.51 & 2566 & 8 & +10 \\
\hline \multicolumn{12}{|c|}{ Igneous zircon } \\
\hline IR41-30 & 0.68 & -0.035 & 0.457 & 2.9 & 10.30 & 2.9 & 0.1636 & 0.40 & 2493 & 7 & +3 \\
\hline IR41-17 & 0.43 & 0.007 & 0.463 & 2.8 & 10.34 & 2.9 & 0.1621 & 0.56 & 2478 & 10 & +1 \\
\hline IR41-11 & 0.70 & 0.111 & 0.466 & 2.9 & 10.39 & 3.0 & 0.1618 & 0.49 & 2474 & 8 & +0 \\
\hline IR41-10 & 0.65 & 0.026 & 0.454 & 2.8 & 10.11 & 2.9 & 0.1615 & 0.73 & 2471 & 12 & +3 \\
\hline IR41-42 & 0.66 & 0.075 & 0.460 & 2.9 & 10.23 & 2.9 & 0.1612 & 0.31 & 2468 & 5 & +1 \\
\hline IR41-26 & 0.43 & 0.064 & 0.445 & 3.0 & 9.87 & 3.0 & 0.1610 & 0.42 & 2466 & 7 & +5 \\
\hline IR41-61 & 0.48 & 0.038 & 0.459 & 2.9 & 10.19 & 3.3 & 0.1609 & 1.55 & 2465 & 26 & +1 \\
\hline IR41-12 & 0.50 & 0.021 & 0.475 & 3.0 & 10.53 & 3.0 & 0.1606 & 0.29 & 2462 & 5 & -2 \\
\hline IR41-58 & 0.29 & 0.069 & 0.451 & 2.9 & 9.98 & 3.1 & 0.1606 & 0.98 & 2462 & 17 & +3 \\
\hline IR41-06 & 0.69 & 0.097 & 0.473 & 2.8 & 10.45 & 2.9 & 0.1603 & 0.29 & 2458 & 5 & -2 \\
\hline IR41-37 & 0.45 & 0.081 & 0.448 & 3.0 & 9.87 & 3.0 & 0.1599 & 0.33 & 2454 & 6 & +3 \\
\hline IR41-08 & 0.40 & -0.007 & 0.447 & 3.2 & 9.83 & 3.8 & 0.1595 & 2.11 & 2450 & 36 & +3 \\
\hline IR41-39 & 0.07 & 0.100 & 0.447 & 3.0 & 9.77 & 3.1 & 0.1587 & 0.59 & 2441 & 10 & +3 \\
\hline IR41-70 & 0.11 & 0.280 & 0.440 & 3.2 & 9.61 & 3.3 & 0.1585 & 0.43 & 2440 & 7 & +4 \\
\hline IR41-47 & 0.45 & 0.039 & 0.448 & 2.8 & 9.76 & 2.8 & 0.1581 & 0.21 & 2435 & 4 & +2 \\
\hline IR41-66 & 0.66 & 0.081 & 0.452 & 2.9 & 9.78 & 2.9 & 0.1569 & 0.30 & 2422 & 5 & +1 \\
\hline IR41-64 & 0.36 & 0.071 & 0.446 & 3.3 & 9.61 & 3.6 & 0.1564 & 1.37 & 2417 & 23 & +2 \\
\hline IR41-69 & 1.24 & 0.126 & 0.447 & 2.9 & 9.63 & 2.9 & 0.1563 & 0.42 & 2416 & 7 & +2 \\
\hline
\end{tabular}


Lane et al. Supplementary Papers

Australian Journal of Earth Sciences (2015) 62, 55-75, http://dx.doi.org/10.1080/08120099.2015.993160

\begin{tabular}{|c|c|c|c|c|c|c|c|c|c|c|c|}
\hline Spot & ${ }^{232} \mathrm{Th} /{ }^{238} \mathrm{U}$ & $\%{ }^{206} \mathrm{~Pb}_{\mathrm{c}}$ & $\begin{array}{c}{ }^{206} \mathrm{~Pb}^{* 3} \mathrm{U} \\
{ }^{23} \mathrm{I}\end{array}$ & $\pm \%$ & $\begin{array}{c}{ }^{207} \mathrm{~Pb}^{235} \mathrm{U} \\
\end{array}$ & $\pm \%$ & $\begin{array}{c}{ }^{207} \mathrm{~Pb}^{*} \mathrm{I} \\
{ }^{206} \mathrm{~Pb}^{*}\end{array}$ & $\pm \%$ & $\begin{array}{c}{ }^{207} \mathrm{~Pb} /{ }^{206} \mathrm{~Pb} \\
\text { Age }\end{array}$ & \pm & $\%$ Disc \\
\hline IR41-32 & 0.54 & 0.128 & 0.427 & 2.9 & 8.91 & 2.9 & 0.1514 & 0.49 & 2362 & 8 & +4 \\
\hline IR41-04 & 0.02 & 0.075 & 0.405 & 3.0 & 7.63 & 3.0 & 0.1366 & 0.23 & 2185 & 4 & -0 \\
\hline \multicolumn{12}{|c|}{ Discordant igneous zircon } \\
\hline IR41-34 & 0.21 & 0.080 & 0.415 & 3.3 & 9.28 & 3.3 & 0.1623 & 0.54 & 2480 & 9 & +12 \\
\hline IR41-29 & 0.52 & 0.102 & 0.518 & 2.9 & 11.57 & 2.9 & 0.1621 & 0.24 & 2477 & 4 & -11 \\
\hline IR41-01 & 0.33 & 0.003 & 0.511 & 2.9 & 11.40 & 2.9 & 0.1619 & 0.30 & 2475 & 5 & -9 \\
\hline IR41-19 & 0.76 & 0.120 & 0.421 & 2.9 & 9.35 & 3.1 & 0.1609 & 1.12 & 2465 & 19 & +10 \\
\hline IR41-57 & 0.58 & 0.064 & 0.432 & 3.0 & 9.56 & 3.0 & 0.1604 & 0.33 & 2460 & 6 & +7 \\
\hline IR41-18 & 0.36 & 0.033 & 0.568 & 2.9 & 12.49 & 2.9 & 0.1595 & 0.44 & 2450 & 7 & -23 \\
\hline IR41-05 & 0.75 & 1.052 & 0.526 & 3.2 & 11.46 & 4.6 & 0.1581 & 3.21 & 2435 & 54 & -14 \\
\hline IR41-27 & 0.41 & 0.644 & 0.393 & 2.9 & 8.55 & 2.9 & 0.1579 & 0.46 & 2433 & 8 & +14 \\
\hline IR41-03 & 0.54 & 0.082 & 0.410 & 2.9 & 8.86 & 3.0 & 0.1568 & 0.30 & 2422 & 5 & +10 \\
\hline IR41-44 & 0.14 & 0.034 & 0.410 & 2.8 & 8.79 & 3.1 & 0.1553 & 1.24 & 2406 & 21 & +9 \\
\hline IR41-33 & 0.23 & 0.524 & 0.353 & 3.1 & 7.49 & 3.1 & 0.1539 & 0.39 & 2389 & 7 & +21 \\
\hline IR41-53 & 0.28 & 0.122 & 0.374 & 3.0 & 7.89 & 3.0 & 0.1529 & 0.43 & 2378 & 7 & +16 \\
\hline IR41-45 & 0.12 & 0.050 & 0.393 & 3.1 & 8.27 & 3.1 & 0.1526 & 0.24 & 2375 & 4 & +12 \\
\hline IR41-52 & 0.42 & 0.131 & 0.385 & 4.1 & 8.05 & 4.2 & 0.1516 & 0.78 & 2364 & 13 & +13 \\
\hline IR41-38 & 0.40 & 0.015 & 0.484 & 7.5 & 10.08 & 8.7 & 0.1511 & 4.44 & 2358 & 76 & -10 \\
\hline IR41-56 & 1.01 & 0.026 & 0.524 & 2.8 & 10.92 & 2.8 & 0.1510 & 0.18 & 2357 & 3 & -19 \\
\hline IR41-50 & 0.84 & 0.227 & 0.389 & 2.8 & 8.10 & 2.9 & 0.1508 & 0.34 & 2355 & 6 & +12 \\
\hline IR41-36 & 0.24 & 0.429 & 0.385 & 2.8 & 7.97 & 2.9 & 0.1503 & 0.64 & 2349 & 11 & +13 \\
\hline IR41-21 & 0.13 & 0.063 & 0.397 & 2.9 & 8.14 & 2.9 & 0.1487 & 0.20 & 2331 & 3 & +9 \\
\hline IR41-68 & 0.43 & 0.014 & 0.363 & 3.0 & 7.44 & 3.0 & 0.1485 & 0.23 & 2329 & 4 & +16 \\
\hline IR41-40 & 0.32 & 0.214 & 0.350 & 2.8 & 7.13 & 2.8 & 0.1477 & 0.23 & 2320 & 4 & +19 \\
\hline IR41-24 & 0.07 & 0.449 & 0.358 & 2.8 & 7.26 & 2.9 & 0.1471 & 0.49 & 2313 & 8 & +17 \\
\hline IR41-15 & 0.51 & 0.049 & 0.296 & 3.4 & 6.00 & 3.5 & 0.1470 & 0.84 & 2312 & 14 & +31 \\
\hline IR41-60 & 0.14 & 0.246 & 0.308 & 3.1 & 6.05 & 3.2 & 0.1426 & 0.77 & 2259 & 13 & +27 \\
\hline IR41-65 & 0.35 & 0.371 & 0.228 & 3.0 & 4.22 & 3.0 & 0.1344 & 0.27 & 2156 & 5 & +43 \\
\hline IR41-31 & 0.16 & 0.170 & 0.211 & 3.9 & 3.73 & 3.9 & 0.1279 & 0.34 & 2069 & 6 & +44 \\
\hline IR41-63 & 0.30 & 0.285 & 0.231 & 3.1 & 4.07 & 3.2 & 0.1278 & 0.58 & 2067 & 10 & +39 \\
\hline IR41-59 & 0.04 & 0.695 & 0.302 & 3.3 & 5.19 & 3.4 & 0.1246 & 0.56 & 2023 & 10 & +18 \\
\hline IR41-28 & 0.03 & 1.365 & 0.338 & 2.8 & 5.68 & 2.9 & 0.1218 & 0.65 & 1983 & 12 & +6 \\
\hline
\end{tabular}


Lane et al. Supplementary Papers

Australian Journal of Earth Sciences (2015) 62, 55-75, http://dx.doi.org/10.1080/08120099.2015.993160

\begin{tabular}{|c|c|c|c|c|c|c|c|c|c|c|c|}
\hline Spot & ${ }^{232} \mathrm{Th} /{ }^{238} \mathrm{U}$ & $\%{ }^{206} \mathrm{~Pb}_{\mathrm{c}}$ & $\begin{array}{c}{ }^{206} \mathrm{~Pb}^{*} \mathrm{I} \\
{ }^{238} \mathrm{U}\end{array}$ & $\pm \%$ & ${ }^{207} \mathrm{~Pb}^{235} \mathrm{U}$ & $\pm \%$ & $\begin{array}{l}{ }^{207} \mathrm{~Pb}^{*} \mathrm{I} \\
{ }^{206} \mathrm{~Pb}^{*}\end{array}$ & $\pm \%$ & $\begin{array}{c}{ }^{207} \mathrm{~Pb} /{ }^{206} \mathrm{~Pb} \\
\mathrm{Age}\end{array}$ & \pm & $\%$ Disc \\
\hline \multicolumn{12}{|c|}{ Metamorphic rims } \\
\hline IR41-49 & 0.03 & 0.064 & 0.406 & 3.4 & 7.90 & 3.4 & 0.1412 & 0.28 & 2242 & 5 & +2 \\
\hline IR41-46 & 0.05 & 0.043 & 0.298 & 2.8 & 4.34 & 2.9 & 0.1057 & 0.56 & 1727 & 10 & +3 \\
\hline IR41-55 & 0.05 & 0.019 & 0.300 & 3.0 & 4.37 & 3.0 & 0.1057 & 0.55 & 1727 & 10 & +2 \\
\hline \multicolumn{12}{|c|}{ Discordant metamorphic zircon } \\
\hline IR41-43 & 0.02 & 0.231 & 0.356 & 2.8 & 6.86 & 2.8 & 0.1396 & 0.34 & 2222 & 6 & +13 \\
\hline IR41-22 & 0.12 & 0.097 & 0.343 & 2.9 & 6.18 & 2.9 & 0.1306 & 0.35 & 2106 & 6 & +11 \\
\hline IR41-51 & 0.03 & 0.240 & 0.315 & 2.8 & 5.02 & 2.9 & 0.1156 & 0.37 & 1890 & 7 & +8 \\
\hline \multicolumn{12}{|l|}{ High Pbc } \\
\hline IR41-23 & 0.32 & 0.981 & 0.452 & 2.8 & 9.70 & 2.9 & 0.1557 & 0.63 & 2410 & 11 & +0 \\
\hline IR41-02 & 0.01 & 0.880 & 0.320 & 3.0 & 4.74 & 3.3 & 0.1073 & 1.47 & 1755 & 27 & -2 \\
\hline $\begin{array}{l}\text { IR41-54 (not } \\
\text { zircon) }\end{array}$ & $-1.5 E+4$ & 0.024 & $-1.8 E+6$ & 128.0 & $-5.0 E+4$ & 130.5 & 0.0002 & 25 & - & - & - \\
\hline
\end{tabular}

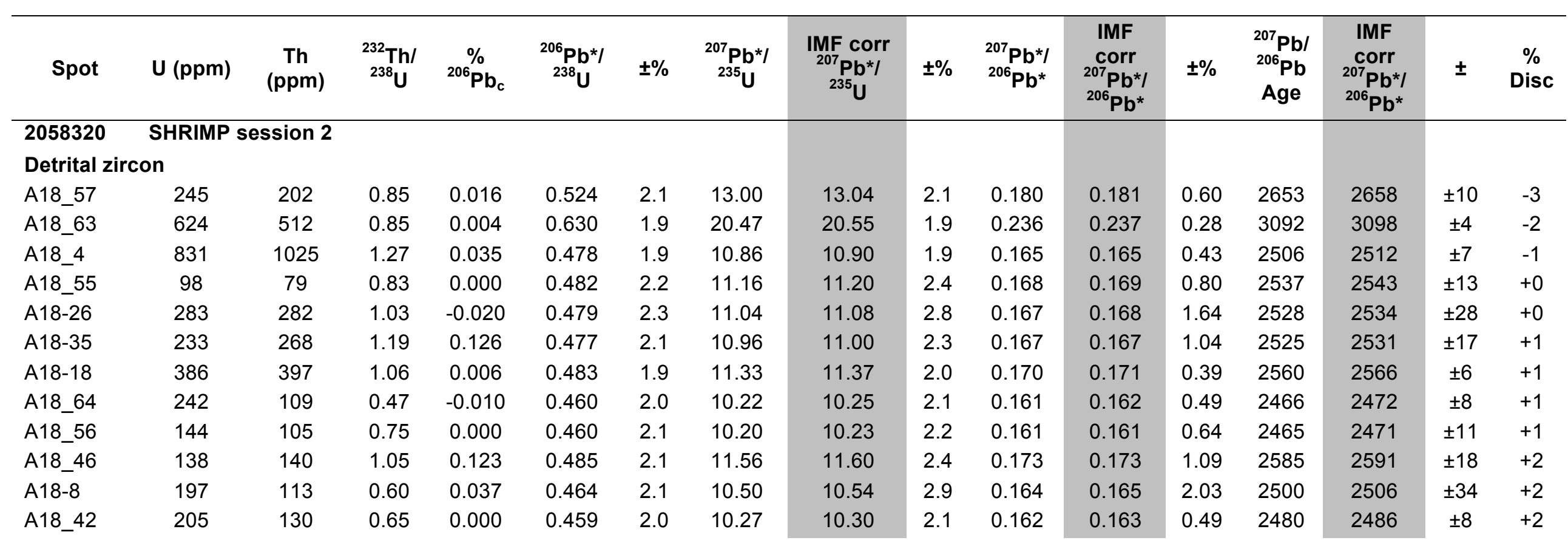




\begin{tabular}{|c|c|c|c|c|c|c|c|c|c|c|c|c|c|c|c|c|}
\hline Spot & U (ppm) & $\begin{array}{c}\text { Th } \\
\text { (ppm) }\end{array}$ & $\begin{array}{c}{ }^{232} \mathrm{Th} / \\
{ }^{238} \mathrm{U}\end{array}$ & $\begin{array}{c}\% \\
{ }^{206} \mathrm{~Pb}_{\mathrm{c}}\end{array}$ & ${ }^{206} \mathrm{~Pb}^{238} \mathrm{U}$ & $\pm \%$ & ${ }^{207} \mathrm{~Pb}^{\star 25} \mathrm{U}$ & $\begin{array}{l}\text { IMF corr } \\
{ }^{207} \mathrm{~Pb}^{*} I \\
{ }^{235} \mathrm{U}\end{array}$ & $\pm \%$ & $\begin{array}{l}{ }^{207} \mathrm{~Pb}^{\star} / \\
{ }^{206} \mathrm{~Pb}^{*}\end{array}$ & $\begin{array}{c}\text { IMF } \\
\text { corr } \\
{ }^{207} \mathrm{~Pb}^{\star /} / \\
{ }^{206} \mathrm{~Pb}^{*}\end{array}$ & $\pm \%$ & $\begin{array}{c}{ }^{207} \mathrm{~Pb} / \\
{ }^{206} \mathrm{~Pb} \\
\mathrm{Age}\end{array}$ & $\begin{array}{c}\text { IMF } \\
\text { corr } \\
{ }^{207} \mathrm{~Pb}^{*} / \\
{ }^{206} \mathrm{~Pb}^{*}\end{array}$ & \pm & $\begin{array}{c}\% \\
\text { Disc }\end{array}$ \\
\hline A18-31 & 382 & 155 & 0.42 & 0.021 & 0.469 & 2.0 & 10.80 & 10.84 & 2.2 & 0.167 & 0.168 & 1.03 & 2529 & 2535 & \pm 17 & +2 \\
\hline A18_59 & 641 & 999 & 1.61 & 0.003 & 0.474 & 1.9 & 11.09 & 11.13 & 1.9 & 0.170 & 0.170 & 0.28 & 2554 & 2560 & \pm 5 & +3 \\
\hline A18-29 & 444 & 193 & 0.45 & 0.014 & 0.467 & 1.9 & 10.77 & 10.81 & 2.2 & 0.167 & 0.168 & 1.02 & 2530 & 2536 & \pm 17 & +3 \\
\hline A18-5 & 379 & 167 & 0.46 & 0.026 & 0.545 & 2.4 & 15.44 & 15.49 & 2.6 & 0.206 & 0.206 & 0.78 & 2871 & 2877 & \pm 13 & +3 \\
\hline A18_39 & 358 & 431 & 1.24 & 0.037 & 0.458 & 1.9 & 10.33 & 10.37 & 2.0 & 0.164 & 0.164 & 0.42 & 2494 & 2500 & \pm 7 & +3 \\
\hline A18_37 & 217 & 122 & 0.58 & 0.069 & 0.608 & 2.0 & 20.52 & 20.60 & 2.2 & 0.245 & 0.246 & 0.80 & 3151 & 3157 & \pm 13 & +3 \\
\hline A18_62 & 182 & 66 & 0.38 & 0.022 & 0.619 & 3.2 & 21.73 & 21.81 & 3.2 & 0.254 & 0.255 & 0.43 & 3213 & 3219 & \pm 7 & +4 \\
\hline A18-23 & 359 & 191 & 0.55 & 0.038 & 0.458 & 2.0 & 10.49 & 10.53 & 2.0 & 0.166 & 0.167 & 0.43 & 2521 & 2527 & \pm 7 & +4 \\
\hline \multicolumn{17}{|c|}{ Discordant detrital zircon } \\
\hline A18-10 & 741 & 88 & 0.12 & 0.027 & 0.475 & 1.9 & 12.62 & 12.67 & 3.3 & 0.193 & 0.193 & 2.67 & 2765 & 2771 & \pm 44 & +11 \\
\hline A18-9 & 1485 & 28 & 0.02 & 0.011 & 0.475 & 2.9 & 12.11 & 12.15 & 3.8 & 0.185 & 0.185 & 2.51 & 2695 & 2701 & \pm 41 & +8 \\
\hline A18_48 & 821 & 20 & 0.03 & 0.021 & 0.471 & 2.6 & 11.78 & 11.82 & 3.1 & 0.182 & 0.182 & 1.71 & 2667 & 2673 & \pm 28 & +8 \\
\hline A18-24 & 156 & 42 & 0.28 & 0.078 & 0.418 & 3.8 & 10.10 & 10.14 & 4.4 & 0.175 & 0.176 & 2.37 & 2610 & 2616 & \pm 39 & +16 \\
\hline A18_49 & 553 & 275 & 0.51 & 0.318 & 0.458 & 1.9 & 10.71 & 10.75 & 2.2 & 0.169 & 0.170 & 1.11 & 2552 & 2558 & \pm 19 & +6 \\
\hline A18-33 & 155 & 65 & 0.43 & 0.117 & 0.395 & 3.8 & 9.12 & 9.15 & 5.0 & 0.168 & 0.168 & 3.18 & 2533 & 2539 & \pm 53 & +18 \\
\hline A18-25 & 93 & 84 & 0.93 & 0.475 & 0.452 & 2.3 & 10.45 & 10.48 & 2.6 & 0.167 & 0.168 & 1.10 & 2532 & 2538 & \pm 18 & +6 \\
\hline A18_36 & 414 & 370 & 0.92 & 0.025 & 0.447 & 1.9 & 10.28 & 10.32 & 1.9 & 0.167 & 0.168 & 0.35 & 2527 & 2533 & \pm 6 & +7 \\
\hline A18_3 & 373 & 245 & 0.68 & 0.101 & 0.452 & 2.7 & 10.32 & 10.36 & 2.9 & 0.166 & 0.166 & 0.84 & 2515 & 2521 & \pm 14 & +5 \\
\hline A18-32 & 390 & 247 & 0.65 & 0.211 & 0.431 & 2.0 & 9.80 & 9.84 & 2.2 & 0.165 & 0.166 & 1.06 & 2509 & 2515 & \pm 18 & +10 \\
\hline A18-28 & 1051 & 618 & 0.61 & 0.033 & 0.444 & 1.9 & 10.04 & 10.08 & 2.4 & 0.164 & 0.165 & 1.40 & 2496 & 2502 & \pm 24 & +6 \\
\hline A18-16 & 394 & 136 & 0.36 & 0.277 & 0.445 & 2.5 & 9.98 & 10.02 & 3.0 & 0.163 & 0.163 & 1.63 & 2482 & 2488 & \pm 27 & +5 \\
\hline A18_60 & 500 & 390 & 0.81 & 0.268 & 0.394 & 1.9 & 8.81 & 8.84 & 1.9 & 0.162 & 0.163 & 0.40 & 2480 & 2486 & \pm 7 & +16 \\
\hline A18_58 & 800 & 473 & 0.61 & 0.027 & 0.439 & 2.3 & 9.75 & 9.78 & 2.3 & 0.161 & 0.162 & 0.31 & 2467 & 2473 & \pm 5 & +6 \\
\hline A18-34 & 444 & 300 & 0.70 & -0.046 & 0.421 & 2.1 & 9.32 & 9.35 & 2.8 & 0.160 & 0.161 & 1.84 & 2461 & 2467 & \pm 31 & +9 \\
\hline A18-11 & 73 & 57 & 0.81 & 0.000 & 0.451 & 2.4 & 9.85 & 9.89 & 4.5 & 0.158 & 0.159 & 3.74 & 2439 & 2445 & \pm 63 & +2 \\
\hline A18_52 & 628 & 442 & 0.73 & 0.079 & 0.365 & 1.9 & 7.96 & 7.99 & 1.9 & 0.158 & 0.159 & 0.32 & 2437 & 2443 & \pm 5 & +21 \\
\hline A18-17 & 1735 & 1683 & 1.00 & 0.127 & 0.342 & 1.8 & 7.21 & 7.24 & 2.1 & 0.153 & 0.154 & 0.89 & 2380 & 2387 & \pm 15 & +23 \\
\hline A18_51 & 778 & 253 & 0.34 & 0.000 & 0.441 & 2.3 & 9.30 & 9.34 & 2.7 & 0.153 & 0.153 & 1.50 & 2379 & 2385 & \pm 26 & +1 \\
\hline A18-6 & 501 & 415 & 0.85 & -0.027 & 0.414 & 2.2 & 8.69 & 8.72 & 2.5 & 0.152 & 0.153 & 1.15 & 2370 & 2376 & \pm 20 & +7 \\
\hline
\end{tabular}




\begin{tabular}{|c|c|c|c|c|c|c|c|c|c|c|c|c|c|c|c|c|}
\hline Spot & U (ppm) & $\begin{array}{c}\text { Th } \\
\text { (ppm) }\end{array}$ & ${ }^{238} \mathrm{Uh} /$ & $\begin{array}{c}\% \\
{ }^{206} \mathrm{~Pb}_{\mathrm{c}}\end{array}$ & ${ }^{206} \mathrm{~Pb}^{238} \mathrm{U}$ & $\pm \%$ & $\begin{array}{c}{ }^{207} \mathrm{~Pb}^{*} \mathrm{I} \\
{ }^{235} \mathrm{U}\end{array}$ & $\begin{array}{c}\text { IMF corr } \\
{ }^{207} \mathrm{~Pb}^{*} \\
{ }^{235} \mathrm{U}\end{array}$ & $\pm \%$ & $\begin{array}{c}{ }^{207} \mathrm{~Pb}^{\star} \mathrm{I} \\
{ }^{206} \mathrm{~Pb}^{\star}\end{array}$ & $\begin{array}{c}\text { IMF } \\
\text { corr } \\
{ }^{207} \mathrm{~Pb}^{\star} / \\
{ }^{206} \mathrm{~Pb}^{*}\end{array}$ & $\pm \%$ & $\begin{array}{c}{ }^{207} \mathrm{~Pb} / \\
{ }^{206} \mathrm{~Pb} \\
\text { Age }\end{array}$ & $\begin{array}{c}\text { IMF } \\
\text { corr } \\
{ }^{207} \mathrm{~Pb}^{*} / \\
{ }^{206} \mathrm{~Pb}^{*}\end{array}$ & \pm & $\begin{array}{c}\% \\
\text { Disc }\end{array}$ \\
\hline A18-20 & 389 & 57 & 0.15 & -0.028 & 0.403 & 2.4 & 8.36 & 8.39 & 2.7 & 0.150 & 0.151 & 1.30 & 2350 & 2357 & \pm 22 & +8 \\
\hline A18-21 & 376 & 69 & 0.19 & 0.000 & 0.389 & 2.0 & 7.73 & 7.76 & 2.4 & 0.144 & 0.145 & 1.41 & 2277 & 2283 & \pm 24 & +8 \\
\hline A18_53 & 176 & 129 & 0.76 & 0.000 & 0.392 & 2.1 & 7.77 & 7.79 & 2.4 & 0.144 & 0.144 & 1.14 & 2274 & 2280 & \pm 20 & +7 \\
\hline A18_2 & 162 & 98 & 0.62 & 0.038 & 0.376 & 2.1 & 7.41 & 7.43 & 3.5 & 0.143 & 0.143 & 2.85 & 2261 & 2267 & \pm 49 & +10 \\
\hline A18_50 & 843 & 229 & 0.28 & 0.072 & 0.291 & 3.1 & 5.72 & 5.74 & 3.6 & 0.142 & 0.143 & 1.81 & 2256 & 2263 & \pm 31 & +31 \\
\hline A18_1 & 571 & 24 & 0.04 & 0.259 & 0.314 & 5.5 & 6.11 & 6.13 & 5.6 & 0.141 & 0.142 & 1.39 & 2240 & 2246 & \pm 24 & +24 \\
\hline A18_44 & 1077 & 734 & 0.70 & 0.097 & 0.257 & 2.0 & 4.97 & 4.99 & 2.4 & 0.140 & 0.141 & 1.29 & 2230 & 2236 & \pm 22 & +38 \\
\hline A18-12 & 574 & 32 & 0.06 & 0.011 & 0.360 & 1.9 & 6.72 & 6.74 & 1.9 & 0.135 & 0.136 & 0.39 & 2165 & 2172 & \pm 7 & +10 \\
\hline \multicolumn{17}{|c|}{ Metamorphic zircon } \\
\hline A18-27 & 525 & 52 & 0.10 & 0.011 & 0.462 & 1.9 & 9.94 & 9.97 & 4.4 & 0.156 & 0.157 & 3.99 & 2414 & 2420 & \pm 68 & -2 \\
\hline A18_38 & 569 & 10 & 0.02 & 0.248 & 0.471 & 2.8 & 10.01 & 10.05 & 3.7 & 0.154 & 0.155 & 2.47 & 2394 & 2400 & \pm 42 & -5 \\
\hline A18-15 & 527 & 157 & 0.31 & 0.024 & 0.430 & 9.2 & 9.09 & 9.12 & 9.3 & 0.153 & 0.154 & 1.18 & 2384 & 2390 & \pm 20 & +4 \\
\hline A18-19 & 458 & 41 & 0.09 & 0.006 & 0.439 & 1.9 & 9.00 & 9.03 & 4.0 & 0.149 & 0.149 & 3.55 & 2331 & 2338 & \pm 61 & -1 \\
\hline A18_41 & 476 & 31 & 0.07 & 0.015 & 0.432 & 3.3 & 8.76 & 8.79 & 4.0 & 0.147 & 0.148 & 2.28 & 2311 & 2318 & \pm 39 & -0 \\
\hline A18_45 & 625 & 4 & 0.01 & 0.016 & 0.410 & 2.4 & 8.31 & 8.34 & 5.2 & 0.147 & 0.147 & 4.56 & 2311 & 2317 & \pm 78 & +5 \\
\hline A18_54 & 807 & 12 & 0.01 & 0.015 & 0.443 & 1.9 & 8.92 & 8.96 & 5.5 & 0.146 & 0.146 & 5.15 & 2299 & 2305 & \pm 88 & -3 \\
\hline A18_65 & 587 & 7 & 0.01 & 0.005 & 0.405 & 4.2 & 8.07 & 8.10 & 4.8 & 0.145 & 0.145 & 2.43 & 2283 & 2289 & \pm 42 & +5 \\
\hline A18_47 & 708 & 14 & 0.02 & 0.034 & 0.424 & 2.7 & 8.43 & 8.46 & 3.1 & 0.144 & 0.145 & 1.45 & 2279 & 2286 & \pm 25 & +0 \\
\hline \multicolumn{17}{|c|}{ Discordant metamorphic zircon } \\
\hline A18_61 & 404 & 133 & 0.34 & 0.016 & 0.398 & 2.9 & 7.83 & 7.86 & 3.0 & 0.143 & 0.143 & 0.50 & 2259 & 2265 & \pm 9 & +5 \\
\hline A18-22 & 529 & 13 & 0.03 & 0.138 & 0.415 & 2.3 & 8.88 & 8.91 & 3.9 & 0.155 & 0.156 & 3.18 & 2405 & 2411 & \pm 54 & +8 \\
\hline A18_43 & 365 & 72 & 0.20 & 0.022 & 0.407 & 3.0 & 8.50 & 8.53 & 3.0 & 0.152 & 0.152 & 0.72 & 2364 & 2370 & \pm 12 & +8 \\
\hline A18-7 & 668 & 6 & 0.01 & 0.004 & 0.398 & 2.7 & 8.21 & 8.24 & 3.4 & 0.150 & 0.150 & 2.11 & 2342 & 2348 & \pm 36 & +9 \\
\hline A18-30 & 496 & 64 & 0.13 & 0.018 & 0.360 & 2.1 & 6.53 & 6.55 & 3.2 & 0.131 & 0.132 & 2.42 & 2118 & 2124 & \pm 42 & +7 \\
\hline \multicolumn{17}{|c|}{ High Pbc } \\
\hline A18-13 & 180 & 109 & 0.62 & 2.301 & 0.409 & 2.5 & 10.40 & 10.44 & 3.6 & 0.185 & 0.185 & 2.57 & 2695 & 2701 & \pm 43 & +21 \\
\hline A18_40 & 396 & 308 & 0.80 & 2.675 & 0.460 & 1.9 & 11.46 & 11.50 & 2.1 & 0.181 & 0.181 & 0.74 & 2658 & 2664 & \pm 12 & +10 \\
\hline A18-14 & 201 & 117 & 0.60 & 0.784 & 0.416 & 2.1 & 9.33 & 9.36 & 3.1 & 0.163 & 0.163 & 2.32 & 2484 & 2490 & \pm 39 & +12 \\
\hline
\end{tabular}




\begin{tabular}{|c|c|c|c|c|c|c|c|c|c|c|c|c|c|c|c|c|}
\hline Spot & U (ppm) & $\begin{array}{c}\text { Th } \\
\text { (ppm) }\end{array}$ & ${ }^{232} \mathrm{Th} /$ & $\begin{array}{c}\% \\
{ }^{206} \mathrm{~Pb}_{\mathrm{c}}\end{array}$ & ${ }^{206}{ }^{238} \mathrm{U}$ *I & $\pm \%$ & ${ }^{2075} \mathrm{~Pb}^{23} \mathrm{U}$ & $\begin{array}{c}\text { IMF corr } \\
{ }^{207} \mathrm{~Pb}^{*} \\
{ }^{235} \mathrm{U}\end{array}$ & $\pm \%$ & $\begin{array}{c}{ }^{207} \mathrm{~Pb}^{*} \mathrm{I} \\
{ }^{206} \mathrm{~Pb}^{*}\end{array}$ & $\begin{array}{c}\text { IMF } \\
\text { corr } \\
{ }^{207} \mathrm{~Pb}^{\star} / \\
{ }^{206} \mathrm{~Pb}^{*}\end{array}$ & $\pm \%$ & $\begin{array}{c}{ }^{207} \mathrm{~Pb} / \\
{ }^{206} \mathrm{~Pb} \\
\text { Age }\end{array}$ & $\begin{array}{c}\text { IMF } \\
\text { corr } \\
{ }^{207} \mathrm{~Pb}^{\star} / \\
{ }^{206} \mathrm{~Pb}^{*}\end{array}$ & \pm & $\begin{array}{c}\% \\
\text { Disc }\end{array}$ \\
\hline \multicolumn{17}{|c|}{$2058328 \quad$ SHRIMP session 2} \\
\hline \multicolumn{17}{|c|}{ Detrital zircon } \\
\hline B23-44 & 416 & 66 & 0.17 & 0.054 & 0.357 & 1.9 & 5.79 & 5.82 & 2.0 & 0.118 & 0.118 & 0.52 & 1923 & 1929 & \pm 9 & -3 \\
\hline B23_37 & 2082 & 1172 & 0.58 & 0.020 & 0.339 & 1.8 & 5.24 & 5.26 & 1.8 & 0.112 & 0.112 & 0.20 & 1832 & 1838 & \pm 4 & -3 \\
\hline B23_6 & 221 & 115 & 0.54 & 0.056 & 0.307 & 2.0 & 4.61 & 4.63 & 2.2 & 0.109 & 0.109 & 0.77 & 1783 & 1790 & \pm 14 & +4 \\
\hline B23-43 & 458 & 246 & 0.56 & -0.019 & 0.321 & 1.9 & 4.82 & 4.84 & 1.9 & 0.109 & 0.109 & 0.43 & 1780 & 1787 & \pm 8 & -1 \\
\hline B23_38 & 223 & 154 & 0.71 & 0.030 & 0.323 & 2.0 & 4.84 & 4.86 & 2.1 & 0.109 & 0.109 & 0.67 & 1780 & 1786 & \pm 12 & -2 \\
\hline B23_4 & 758 & 769 & 1.05 & 0.225 & 0.307 & 1.9 & 4.59 & 4.60 & 1.9 & 0.108 & 0.109 & 0.50 & 1773 & 1779 & \pm 9 & +3 \\
\hline B23_33 & 2035 & 2404 & 1.22 & 0.025 & 0.327 & 1.8 & 4.89 & 4.91 & 1.8 & 0.108 & 0.109 & 0.21 & 1773 & 1779 & \pm 4 & -3 \\
\hline B23-45 & 308 & 165 & 0.55 & 0.045 & 0.304 & 1.9 & 4.54 & 4.56 & 2.0 & 0.108 & 0.109 & 0.59 & 1771 & 1778 & \pm 11 & +4 \\
\hline B23_12 & 299 & 64 & 0.22 & 0.219 & 0.303 & 2.0 & 4.51 & 4.53 & 2.1 & 0.108 & 0.108 & 0.77 & 1767 & 1774 & \pm 14 & +4 \\
\hline B23_7 & 790 & 166 & 0.22 & 0.062 & 0.328 & 1.9 & 4.86 & 4.88 & 2.0 & 0.108 & 0.108 & 0.79 & 1759 & 1766 & \pm 14 & -4 \\
\hline B23-49 & 167 & 119 & 0.74 & -0.026 & 0.327 & 2.5 & 4.85 & 4.86 & 2.6 & 0.108 & 0.108 & 0.87 & 1759 & 1765 & \pm 16 & -4 \\
\hline B23-47 & 240 & 50 & 0.22 & 0.070 & 0.305 & 2.0 & 4.52 & 4.54 & 2.1 & 0.107 & 0.108 & 0.67 & 1756 & 1763 & \pm 12 & +2 \\
\hline B23-50 & 169 & 71 & 0.43 & -0.020 & 0.314 & 2.0 & 4.64 & 4.65 & 2.2 & 0.107 & 0.107 & 0.76 & 1751 & 1757 & \pm 14 & -1 \\
\hline B23_36 & 117 & 77 & 0.68 & 0.143 & 0.316 & 2.1 & 4.66 & 4.68 & 2.4 & 0.107 & 0.107 & 1.04 & 1749 & 1755 & \pm 19 & -1 \\
\hline B23-42 & 602 & 80 & 0.14 & 0.043 & 0.310 & 1.9 & 4.56 & 4.57 & 1.9 & 0.107 & 0.107 & 0.38 & 1745 & 1752 & \pm 7 & +0 \\
\hline B23_3 & 64 & 19 & 0.31 & 0.074 & 0.313 & 2.5 & 4.61 & 4.63 & 3.0 & 0.107 & 0.107 & 1.57 & 1745 & 1752 & \pm 29 & -1 \\
\hline B23_32 & 1236 & 1160 & 0.97 & 0.062 & 0.310 & 1.8 & 4.56 & 4.57 & 1.9 & 0.107 & 0.107 & 0.30 & 1744 & 1750 & \pm 5 & +0 \\
\hline B23_27 & 359 & 130 & 0.37 & 0.142 & 0.304 & 1.9 & 4.47 & 4.48 & 2.0 & 0.107 & 0.107 & 0.62 & 1743 & 1750 & \pm 11 & +2 \\
\hline B23_28 & 556 & 317 & 0.59 & 0.012 & 0.305 & 1.9 & 4.49 & 4.51 & 1.9 & 0.107 & 0.107 & 0.42 & 1743 & 1750 & \pm 8 & +2 \\
\hline B23-48 & 428 & 378 & 0.91 & 0.101 & 0.323 & 1.9 & 4.75 & 4.77 & 2.0 & 0.107 & 0.107 & 0.54 & 1743 & 1749 & \pm 10 & -4 \\
\hline B23_2 & 293 & 53 & 0.19 & -0.015 & 0.302 & 2.0 & 4.44 & 4.46 & 2.1 & 0.107 & 0.107 & 0.67 & 1743 & 1749 & \pm 12 & +3 \\
\hline B23_17 & 268 & 123 & 0.47 & 0.084 & 0.295 & 2.0 & 4.34 & 4.36 & 2.1 & 0.107 & 0.107 & 0.69 & 1742 & 1749 & \pm 13 & +5 \\
\hline B23-39 & 247 & 18 & 0.08 & 0.000 & 0.306 & 2.0 & 4.50 & 4.51 & 2.1 & 0.107 & 0.107 & 0.65 & 1741 & 1747 & \pm 12 & +1 \\
\hline B23_29 & 542 & 96 & 0.18 & 0.081 & 0.315 & 1.9 & 4.63 & 4.64 & 1.9 & 0.106 & 0.107 & 0.44 & 1740 & 1747 & \pm 8 & -2 \\
\hline B23-46 & 467 & 25 & 0.06 & 0.062 & 0.317 & 1.9 & 4.65 & 4.67 & 2.0 & 0.106 & 0.107 & 0.63 & 1736 & 1742 & \pm 12 & -3 \\
\hline B23-41 & 293 & 146 & 0.51 & 0.036 & 0.315 & 1.9 & 4.61 & 4.62 & 2.0 & 0.106 & 0.107 & 0.61 & 1736 & 1742 & \pm 11 & -2 \\
\hline B23_15 & 713 & 26 & 0.04 & 0.024 & 0.308 & 1.9 & 4.50 & 4.51 & 2.0 & 0.106 & 0.106 & 0.44 & 1727 & 1734 & \pm 8 & -0 \\
\hline
\end{tabular}




\begin{tabular}{|c|c|c|c|c|c|c|c|c|c|c|c|c|c|c|c|c|}
\hline Spot & U (ppm) & $\begin{array}{c}\text { Th } \\
\text { (ppm) }\end{array}$ & ${ }^{238} \mathrm{U} /$ & $\begin{array}{c}\% \\
{ }^{206} \mathrm{~Pb}_{\mathrm{c}}\end{array}$ & ${ }^{206}{ }^{238} \mathrm{U}$ *I & $\pm \%$ & ${ }^{2075} \mathrm{~Pb}^{23} \mathrm{U}$ & $\begin{array}{c}\text { IMF corr } \\
{ }_{207} \mathrm{~Pb}^{*} \text { I } \\
{ }^{235} \mathrm{U}\end{array}$ & $\pm \%$ & $\begin{array}{c}{ }^{207} \mathrm{~Pb}^{\star} \mathrm{I} \\
{ }^{206} \mathrm{~Pb}^{*}\end{array}$ & $\begin{array}{c}\text { IMF } \\
\text { corr } \\
{ }^{207} \mathrm{~Pb}^{\star} / \\
{ }^{206} \mathrm{~Pb}^{*}\end{array}$ & $\pm \%$ & $\begin{array}{c}{ }^{207} \mathrm{~Pb} / \\
{ }^{206} \mathrm{~Pb} \\
\text { Age }\end{array}$ & $\begin{array}{c}\text { IMF } \\
\text { corr } \\
{ }^{207} \mathrm{~Pb}^{\star} / \\
{ }^{206} \mathrm{~Pb}^{*}\end{array}$ & \pm & $\begin{array}{c}\% \\
\text { Disc }\end{array}$ \\
\hline B23_31 & 953 & 40 & 0.04 & 0.106 & 0.316 & 1.9 & 4.55 & 4.57 & 1.9 & 0.105 & 0.105 & 0.39 & 1707 & 1713 & \pm 7 & -4 \\
\hline \multicolumn{17}{|c|}{ Discordant cores } \\
\hline B23_8 & 1655 & 43 & 0.03 & 0.015 & 0.344 & 1.8 & 5.29 & 5.31 & 1.9 & 0.111 & 0.112 & 0.24 & 1822 & 1829 & \pm 4 & -5 \\
\hline B23_14 & 682 & 27 & 0.04 & 0.084 & 0.297 & 1.9 & 4.45 & 4.47 & 1.9 & 0.109 & 0.109 & 0.48 & 1780 & 1786 & \pm 9 & +7 \\
\hline B23_13 & 174 & 66 & 0.39 & 0.358 & 0.299 & 2.1 & 4.47 & 4.49 & 2.8 & 0.108 & 0.109 & 1.89 & 1773 & 1780 & \pm 34 & +6 \\
\hline B23_5 & 788 & 31 & 0.04 & 0.213 & 0.288 & 1.9 & 4.28 & 4.30 & 1.9 & 0.108 & 0.108 & 0.47 & 1765 & 1772 & \pm 9 & +9 \\
\hline B23_1 & 393 & 36 & 0.10 & 0.081 & 0.265 & 2.1 & 3.91 & 3.93 & 2.2 & 0.107 & 0.108 & 0.62 & 1754 & 1761 & \pm 11 & +15 \\
\hline B23-40 & 872 & 62 & 0.07 & 0.262 & 0.282 & 1.9 & 4.15 & 4.17 & 1.9 & 0.107 & 0.107 & 0.45 & 1746 & 1752 & \pm 8 & +9 \\
\hline B23_21 & 1746 & 100 & 0.06 & 0.043 & 0.327 & 2.0 & 4.77 & 4.79 & 2.1 & 0.106 & 0.106 & 0.47 & 1729 & 1736 & \pm 9 & -6 \\
\hline B23_35 & 256 & 119 & 0.48 & 0.115 & 0.329 & 2.0 & 4.75 & 4.77 & 2.1 & 0.105 & 0.105 & 0.65 & 1711 & 1718 & \pm 12 & -8 \\
\hline B23_20 & 232 & 78 & 0.35 & 0.023 & 0.265 & 2.5 & 3.71 & 3.73 & 6.6 & 0.102 & 0.102 & 6.16 & 1655 & 1662 & \pm 114 & +10 \\
\hline B23_34 & 1244 & 111 & 0.09 & 0.279 & 0.250 & 1.9 & 3.48 & 3.50 & 1.9 & 0.101 & 0.102 & 0.42 & 1645 & 1652 & \pm 8 & +14 \\
\hline \multicolumn{17}{|c|}{ Metamorphic zircon rims } \\
\hline B23_16 & 190 & 22 & 0.12 & -0.083 & 0.313 & 2.1 & 4.56 & 4.58 & 2.2 & 0.106 & 0.106 & 0.83 & 1729 & 1736 & \pm 15 & -2 \\
\hline B23_19 & 187 & 22 & 0.12 & -0.021 & 0.315 & 2.1 & 4.57 & 4.59 & 2.2 & 0.105 & 0.106 & 0.78 & 1719 & 1726 & \pm 14 & -3 \\
\hline B23_30 & 41 & 14 & 0.36 & 0.156 & 0.313 & 2.7 & 4.51 & 4.53 & 3.3 & 0.104 & 0.105 & 1.90 & 1704 & 1711 & \pm 35 & -4 \\
\hline \multicolumn{17}{|c|}{ Metamorphic zircon } \\
\hline B23_10 & 221 & 12 & 0.06 & -0.017 & 0.307 & 2.0 & 4.49 & 4.50 & 2.1 & 0.106 & 0.106 & 0.71 & 1730 & 1737 & \pm 13 & +0 \\
\hline B23_11 & 322 & 11 & 0.04 & 0.210 & 0.302 & 3.3 & 4.40 & 4.42 & 3.3 & 0.106 & 0.106 & 0.67 & 1726 & 1733 & \pm 12 & +2 \\
\hline B23_18 & 118 & 72 & 0.63 & 0.070 & 0.308 & 2.2 & 4.43 & 4.45 & 2.4 & 0.105 & 0.105 & 1.08 & 1706 & 1713 & \pm 20 & -2 \\
\hline B23_9 & 131 & 20 & 0.16 & 0.064 & 0.310 & 2.2 & 4.43 & 4.45 & 2.4 & 0.104 & 0.104 & 1.04 & 1691 & 1698 & \pm 19 & -3 \\
\hline 2058318 & SHRIMP & ssion 2 & & & & & & & & & & & & & & \\
\hline \multicolumn{17}{|c|}{ Detrital zircon } \\
\hline CO3-10 & 632 & 530 & 0.87 & 0.01 & 0.325 & 1.9 & 4.85 & 4.86 & 1.9 & 0.108 & 0.109 & 0.36 & 1770 & 1777 & \pm 7 & -3 \\
\hline CO3-17 & 431 & 313 & 0.75 & 0.16 & 0.311 & 1.9 & 4.62 & 4.64 & 2.0 & 0.108 & 0.108 & 0.51 & 1764 & 1771 & \pm 9 & +1 \\
\hline CO3-15 & 665 & 419 & 0.65 & 0.08 & 0.301 & 1.9 & 4.47 & 4.48 & 1.9 & 0.108 & 0.108 & 0.38 & 1761 & 1767 & \pm 7 & +4 \\
\hline co3-6 & 365 & 183 & 0.52 & 0.05 & 0.316 & 1.9 & 4.68 & 4.69 & 2.0 & 0.107 & 0.108 & 0.48 & 1755 & 1761 & \pm 9 & -1 \\
\hline CO3-1 & 158 & 18 & 0.12 & 0.04 & 0.314 & 2.1 & 4.63 & 4.64 & 2.2 & 0.107 & 0.107 & 0.79 & 1747 & 1754 & \pm 14 & -1 \\
\hline CO3-2 & 194 & 106 & 0.56 & 0.09 & 0.307 & 2.0 & 4.52 & 4.54 & 2.1 & 0.107 & 0.107 & 0.77 & 1745 & 1751 & \pm 14 & +1 \\
\hline
\end{tabular}




\begin{tabular}{|c|c|c|c|c|c|c|c|c|c|c|c|c|c|c|c|c|}
\hline Spot & U (ppm) & $\begin{array}{c}\text { Th } \\
\text { (ppm) }\end{array}$ & ${ }^{238} \mathrm{Uh} /$ & $\begin{array}{c}\% \\
{ }^{206} \mathrm{~Pb}_{\mathrm{c}}\end{array}$ & ${ }^{206}{ }^{238} \mathrm{U}$. I & $\pm \%$ & ${ }^{207} \mathrm{~Pb}^{235} \mathrm{U}$ & $\begin{array}{c}\text { IMF corr } \\
{ }^{207} \mathrm{~Pb}^{*} \text { I } \\
{ }^{235} \mathrm{U}\end{array}$ & $\pm \%$ & $\begin{array}{c}{ }^{207} \mathrm{~Pb}^{*} \mathrm{I} \\
{ }^{206} \mathrm{~Pb}^{*}\end{array}$ & $\begin{array}{c}\text { IMF } \\
\text { Corr } \\
{ }^{207} \mathrm{~Pb}^{*} \text { I } \\
{ }^{206} \mathrm{~Pb}^{*}\end{array}$ & $\pm \%$ & $\begin{array}{c}{ }^{207} \mathrm{~Pb} / \\
{ }^{206} \mathrm{~Pb} \\
\text { Age }\end{array}$ & $\begin{array}{c}\text { IMF } \\
\text { corr } \\
{ }^{207} \mathrm{~Pb}^{\star} / \\
{ }^{206} \mathrm{~Pb}^{*}\end{array}$ & \pm & $\begin{array}{c}\% \\
\text { Disc }\end{array}$ \\
\hline CO3-13 & 240 & 106 & 0.46 & 0.06 & 0.312 & 2.0 & 4.59 & 4.60 & 2.1 & 0.107 & 0.107 & 0.65 & 1744 & 1751 & \pm 12 & -0 \\
\hline CO3-9 & 204 & 103 & 0.53 & 0.09 & 0.309 & 2.0 & 4.49 & 4.51 & 2.2 & 0.105 & 0.106 & 0.78 & 1722 & 1729 & \pm 14 & -1 \\
\hline CO3-8 & 187 & 109 & 0.60 & 0.05 & 0.315 & 2.0 & 4.58 & 4.59 & 2.1 & 0.105 & 0.106 & 0.74 & 1720 & 1726 & \pm 14 & -3 \\
\hline \multicolumn{17}{|c|}{ Metamorphic zircon rims } \\
\hline CO3-4 & 88 & 11 & 0.13 & 0.08 & 0.314 & 2.4 & 4.60 & 4.61 & 3.0 & 0.106 & 0.107 & 1.68 & 1735 & 1742 & \pm 31 & -2 \\
\hline CO3-12 & 927 & 125 & 0.14 & 0.07 & 0.292 & 1.9 & 4.24 & 4.26 & 1.9 & 0.105 & 0.106 & 0.34 & 1720 & 1727 & \pm 6 & +4 \\
\hline \multicolumn{17}{|c|}{ Discordant zircon } \\
\hline CO3-11 & 391 & 126 & 0.33 & 0.02 & 0.372 & 1.9 & 5.98 & 6.00 & 2.0 & 0.117 & 0.117 & 0.38 & 1904 & 1910 & \pm 7 & -8 \\
\hline CO3-5 & 292 & 146 & 0.52 & 0.18 & 0.259 & 2.0 & 3.99 & 4.01 & 2.1 & 0.112 & 0.112 & 0.73 & 1829 & 1836 & \pm 13 & +21 \\
\hline CO3-7 & 222 & 177 & 0.82 & 0.03 & 0.277 & 2.0 & 4.21 & 4.22 & 2.1 & 0.110 & 0.111 & 0.70 & 1802 & 1809 & \pm 13 & +14 \\
\hline co3-3 & 671 & 231 & 0.36 & 0.03 & 0.334 & 1.9 & 4.96 & 4.98 & 1.9 & 0.108 & 0.108 & 0.39 & 1758 & 1765 & \pm 7 & -7 \\
\hline CO3-16 & 265 & 74 & 0.29 & 0.16 & 0.262 & 2.3 & 3.84 & 3.85 & 2.6 & 0.106 & 0.107 & 1.23 & 1738 & 1744 & \pm 23 & +15 \\
\hline
\end{tabular}

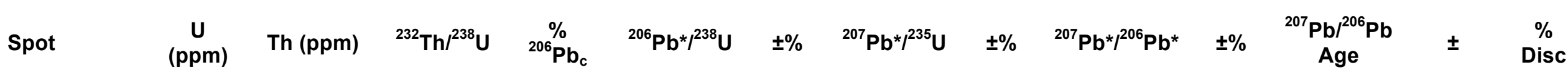

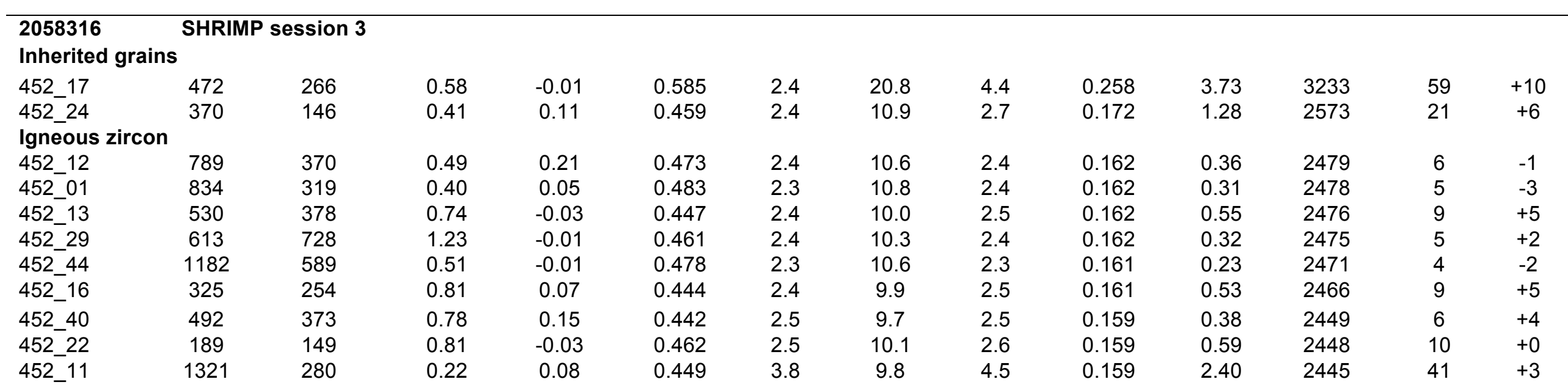


Lane et al. Supplementary Papers

Australian Journal of Earth Sciences (2015) 62, 55-75, http://dx.doi.org/10.1080/08120099.2015.993160

\begin{tabular}{|c|c|c|c|c|c|c|c|c|c|c|c|c|c|}
\hline Spot & $\underset{(p p m)}{U}$ & Th (ppm) & ${ }^{232} \mathrm{Th} /{ }^{238} \mathrm{U}$ & $\begin{array}{c}\% \\
{ }^{206} \mathrm{~Pb}_{\mathrm{c}}\end{array}$ & ${ }^{206} \mathrm{~Pb}^{\star} I^{238} \mathrm{U}$ & $\pm \%$ & $\left.{ }^{207} \mathrm{~Pb}{ }^{\star}\right|^{235} \mathrm{U}$ & $\pm \%$ & ${ }^{207} \mathrm{~Pb}^{\star} I^{206} \mathrm{~Pb}^{*}$ & $\pm \%$ & $\begin{array}{c}{ }^{207} \mathrm{~Pb} /{ }^{206} \mathrm{~Pb} \\
\text { Age }\end{array}$ & \pm & $\begin{array}{c}\% \\
\text { Disc }\end{array}$ \\
\hline $452 \_26$ & 773 & 128 & 0.17 & 0.00 & 0.473 & 2.3 & 10.3 & 2.4 & 0.159 & 0.28 & 2443 & 5 & -3 \\
\hline 452_21 & 2045 & 183 & 0.09 & 0.16 & 0.442 & 2.6 & 9.7 & 2.9 & 0.158 & 1.27 & 2438 & 22 & +4 \\
\hline 452_38 & 1741 & 1907 & 1.13 & 0.04 & 0.466 & 2.3 & 10.1 & 2.3 & 0.158 & 0.20 & 2431 & 3 & -2 \\
\hline 452_39 & 1170 & 476 & 0.42 & 0.00 & 0.449 & 2.3 & 9.7 & 2.3 & 0.156 & 0.23 & 2416 & 4 & +1 \\
\hline 452_25 & 724 & 209 & 0.30 & 0.02 & 0.440 & 2.6 & 9.2 & 2.7 & 0.152 & 0.61 & 2368 & 10 & +1 \\
\hline 452_35 & 1953 & 97 & 0.05 & 0.00 & 0.437 & 2.7 & 9.0 & 2.9 & 0.149 & 1.18 & 2339 & 20 & +0 \\
\hline 452_36 & 3469 & 61 & 0.02 & 0.22 & 0.418 & 2.5 & 8.4 & 2.6 & 0.146 & 0.66 & 2302 & 11 & +3 \\
\hline 452_23 & 250 & 139 & 0.58 & 0.03 & 0.409 & 2.4 & 8.2 & 3.4 & 0.145 & 2.33 & 2291 & 40 & +4 \\
\hline \multicolumn{14}{|c|}{ Discordant igneous zircon } \\
\hline 452_27 & 517 & 187 & 0.37 & 0.30 & 0.396 & 4.3 & 8.8 & 4.3 & 0.162 & 0.53 & 2479 & 9 & +16 \\
\hline 452_30 & 759 & 530 & 0.72 & 0.14 & 0.416 & 2.3 & 9.2 & 2.4 & 0.160 & 0.32 & 2456 & 5 & +10 \\
\hline 452_07 & 545 & 356 & 0.68 & 0.17 & 0.408 & 2.4 & 8.9 & 2.5 & 0.159 & 0.65 & 2443 & 11 & +12 \\
\hline 452_32 & 1108 & 229 & 0.21 & 0.06 & 0.513 & 2.9 & 11.1 & 3.1 & 0.157 & 1.17 & 2423 & 20 & -12 \\
\hline 452_28 & 1100 & 212 & 0.20 & 0.12 & 0.382 & 2.7 & 8.2 & 2.8 & 0.156 & 0.65 & 2408 & 11 & +16 \\
\hline $452 \_46$ & 1208 & 551 & 0.47 & 0.23 & 0.371 & 2.3 & 7.9 & 2.4 & 0.156 & 0.47 & 2407 & 8 & +18 \\
\hline $452 \_48$ & 2307 & 31 & 0.01 & 0.17 & 0.476 & 2.3 & 10.2 & 2.3 & 0.155 & 0.19 & 2403 & 3 & -5 \\
\hline 452_05 & 2206 & 40 & 0.02 & 0.10 & 0.408 & 2.3 & 8.7 & 2.3 & 0.155 & 0.22 & 2402 & 4 & +10 \\
\hline 452_34 & 3398 & 436 & 0.13 & 0.06 & 0.390 & 2.3 & 8.2 & 2.3 & 0.153 & 0.25 & 2376 & 4 & +12 \\
\hline $452 \_43$ & 2486 & 135 & 0.06 & 0.16 & 0.395 & 2.3 & 8.3 & 2.3 & 0.152 & 0.19 & 2364 & 3 & +11 \\
\hline 452_19 & 590 & 97 & 0.17 & 0.05 & 0.362 & 2.4 & 7.4 & 2.5 & 0.148 & 0.78 & 2324 & 13 & +17 \\
\hline $452 \_47$ & 3381 & 198 & 0.06 & 0.26 & 0.333 & 2.4 & 6.7 & 2.4 & 0.146 & 0.28 & 2304 & 5 & +22 \\
\hline 452_08 & 3890 & 632 & 0.17 & 0.22 & 0.365 & 2.3 & 7.3 & 2.3 & 0.146 & 0.28 & 2300 & 5 & +15 \\
\hline 452_03 & 1875 & 573 & 0.32 & 0.54 & 0.320 & 2.4 & 6.3 & 2.4 & 0.144 & 0.55 & 2276 & 9 & +24 \\
\hline 452_33 & 1875 & 497 & 0.27 & 0.11 & 0.333 & 2.8 & 6.6 & 3.1 & 0.144 & 1.24 & 2273 & 21 & +21 \\
\hline 452_37 & 2969 & 363 & 0.13 & 0.38 & 0.308 & 2.3 & 6.1 & 2.3 & 0.142 & 0.23 & 2256 & 4 & +26 \\
\hline $452 \_15$ & 1963 & 220 & 0.12 & 0.16 & 0.259 & 2.3 & 5.0 & 2.3 & 0.139 & 0.29 & 2214 & 5 & +37 \\
\hline $452 \_45$ & 1497 & 163 & 0.11 & 1.11 & 0.260 & 3.2 & 4.9 & 3.3 & 0.137 & 0.55 & 2195 & 10 & +36 \\
\hline $452 \_18$ & 2313 & 33 & 0.01 & 0.12 & 0.261 & 2.9 & 4.9 & 2.9 & 0.136 & 0.26 & 2182 & 5 & +35 \\
\hline $452 \_42$ & 1956 & 97 & 0.05 & 1.66 & 0.262 & 2.3 & 4.9 & 2.5 & 0.136 & 0.79 & 2177 & 14 & +35 \\
\hline 452_20 & 1147 & 843 & 0.76 & 0.30 & 0.254 & 3.3 & 4.7 & 3.4 & 0.135 & 0.75 & 2158 & 13 & +36 \\
\hline 452_31 & 2655 & 30 & 0.01 & 0.56 & 0.426 & 2.3 & 7.9 & 2.4 & 0.134 & 0.75 & 2157 & 13 & -7 \\
\hline 452_02 & 1975 & 53 & 0.03 & 0.89 & 0.273 & 7.3 & 5.1 & 7.4 & 0.134 & 1.53 & 2153 & 27 & +31 \\
\hline 452_04 & 3734 & 60 & 0.02 & 0.06 & 0.273 & 2.5 & 5.0 & 2.5 & 0.132 & 0.19 & 2119 & 3 & +30 \\
\hline 452_09 & 2145 & 1166 & 0.56 & 0.17 & 0.185 & 2.4 & 3.1 & 2.4 & 0.121 & 0.33 & 1964 & 6 & +48 \\
\hline
\end{tabular}


Lane et al. Supplementary Papers

Australian Journal of Earth Sciences (2015) 62, 55-75, http://dx.doi.org/10.1080/08120099.2015.993160

\begin{tabular}{|c|c|c|c|c|c|c|c|c|c|c|c|c|c|}
\hline Spot & $\begin{array}{c}\text { U } \\
(p p m)\end{array}$ & Th (ppm) & ${ }^{232} \mathrm{Th} /{ }^{238} \mathrm{U}$ & $\begin{array}{c}\% \\
{ }^{206} \mathrm{~Pb}_{\mathrm{c}}\end{array}$ & $\left.{ }^{206} \mathrm{~Pb}^{\star}\right|^{238} \mathrm{U}$ & $\pm \%$ & $\left.{ }^{207} \mathrm{~Pb}^{\star}\right|^{235} \mathrm{U}$ & $\pm \%$ & $\left.{ }^{207} \mathrm{~Pb}^{\star}\right|^{206} \mathrm{~Pb}^{*}$ & $\pm \%$ & $\begin{array}{c}{ }^{207} \mathrm{~Pb} /{ }^{206} \mathrm{~Pb} \\
\mathrm{Age}\end{array}$ & \pm & $\begin{array}{c}\% \\
\text { Disc }\end{array}$ \\
\hline $452 \_10$ & 1761 & 54 & 0.03 & 0.81 & 0.156 & 2.3 & 2.1 & 2.5 & 0.099 & 0.93 & 1601 & 17 & +45 \\
\hline \multicolumn{14}{|c|}{ Metamorphic rims } \\
\hline $452 \_41$ & 1750 & 112 & 0.07 & 0.09 & 0.512 & 2.5 & 11.1 & 2.5 & 0.157 & 0.21 & 2420 & 4 & -12 \\
\hline 452_06 & 4219 & 66 & 0.02 & 0.08 & 0.433 & 2.4 & 9.2 & 2.4 & 0.155 & 0.15 & 2401 & 3 & +4 \\
\hline $452-14$ & 2553 & 38 & 0.02 & 0.05 & 0.456 & 2.3 & 9.3 & 2.3 & 0.147 & 0.20 & 2316 & 3 & -6 \\
\hline $205 \overline{8} 317$ & \multicolumn{13}{|c|}{ SHRIMP session 3} \\
\hline \multicolumn{14}{|c|}{ Inherited grains } \\
\hline 424_31 & 855 & 184 & 0.22 & 0.01 & 0.577 & 2.4 & 16.4 & 2.5 & 0.206 & 0.87 & 2872 & 14 & -3 \\
\hline 424_20 & 294 & 50 & 0.17 & -0.03 & 0.523 & 2.5 & 13.2 & 5.0 & 0.183 & 4.30 & 2680 & 71 & -1 \\
\hline 424_36 & 306 & 36 & 0.12 & 0.02 & 0.472 & 3.0 & 11.2 & 7.2 & 0.172 & 6.55 & 2578 & 109 & +4 \\
\hline 424_9 & 402 & 59 & 0.15 & 0.01 & 0.478 & 2.8 & 11.3 & 4.4 & 0.171 & 3.32 & 2567 & 55 & +2 \\
\hline 424_11 & 577 & 372 & 0.67 & 0.01 & 0.482 & 2.4 & 11.3 & 2.4 & 0.170 & 0.34 & 2560 & 6 & +1 \\
\hline 424_4 & 255 & 86 & 0.35 & -0.03 & 0.489 & 3.0 & 11.4 & 3.7 & 0.169 & 2.11 & 2550 & 35 & -1 \\
\hline 424_33 & 335 & 46 & 0.14 & -0.03 & 0.471 & 2.4 & 11.0 & 4.2 & 0.169 & 3.46 & 2544 & 58 & +3 \\
\hline 424_10 & 387 & 44 & 0.12 & 0.04 & 0.478 & 2.4 & 11.0 & 5.0 & 0.167 & 4.39 & 2527 & 74 & +0 \\
\hline 424_17 & 868 & 462 & 0.55 & 0.00 & 0.485 & 2.4 & 11.1 & 2.4 & 0.165 & 0.38 & 2512 & 6 & -2 \\
\hline 424_34 & 403 & 150 & 0.39 & 0.01 & 0.483 & 2.4 & 11.0 & 2.9 & 0.165 & 1.62 & 2507 & 27 & -2 \\
\hline 424_32 & 521 & 65 & 0.13 & 0.01 & 0.468 & 2.6 & 10.6 & 3.2 & 0.164 & 1.87 & 2493 & 32 & +1 \\
\hline \multicolumn{14}{|c|}{ Igneous zircon } \\
\hline $424 \_13$ & 554 & 121 & 0.23 & 0.02 & 0.448 & 2.4 & 10.0 & 2.4 & 0.161 & 0.35 & 2470 & 6 & +4 \\
\hline 424_8 & 392 & 78 & 0.21 & 0.04 & 0.459 & 2.6 & 10.2 & 2.8 & 0.161 & 0.88 & 2465 & 15 & +2 \\
\hline 424_3 & 333 & 62 & 0.19 & -0.01 & 0.481 & 3.3 & 10.6 & 3.8 & 0.160 & 1.93 & 2457 & 33 & -4 \\
\hline 424_15 & 369 & 50 & 0.14 & 0.00 & 0.477 & 2.5 & 10.5 & 2.5 & 0.160 & 0.54 & 2454 & 9 & -3 \\
\hline $424-5$ & 666 & 154 & 0.24 & -0.01 & 0.425 & 2.4 & 8.6 & 2.4 & 0.148 & 0.42 & 2317 & 7 & +2 \\
\hline \multicolumn{14}{|c|}{ Discorordant zircon } \\
\hline 424_16 & 743 & 354 & 0.49 & 0.36 & 0.456 & 2.4 & 12.8 & 2.4 & 0.204 & 0.53 & 2859 & 9 & +18 \\
\hline 424_18 & 688 & 150 & 0.22 & 0.08 & 0.453 & 3.1 & 12.2 & 3.3 & 0.196 & 1.13 & 2795 & 18 & +17 \\
\hline 424_30 & 913 & 305 & 0.35 & 0.02 & 0.571 & 2.4 & 14.4 & 2.6 & 0.183 & 0.92 & 2676 & 15 & -11 \\
\hline 424_2 & 789 & 1053 & 1.38 & 0.27 & 0.433 & 2.4 & 10.6 & 2.5 & 0.178 & 0.53 & 2632 & 9 & +14 \\
\hline 424_1 & 437 & 227 & 0.54 & 0.00 & 0.465 & 2.4 & 11.2 & 2.6 & 0.174 & 0.97 & 2597 & 16 & +6 \\
\hline 424_29 & 851 & 222 & 0.27 & 0.27 & 0.416 & 2.6 & 9.4 & 3.6 & 0.164 & 2.42 & 2502 & 41 & +12 \\
\hline 424_12 & 402 & 414 & 1.06 & -0.01 & 0.498 & 2.4 & 11.2 & 2.5 & 0.163 & 0.47 & 2488 & 8 & -6 \\
\hline 424_26 & 315 & 221 & 0.73 & 0.01 & 0.399 & 2.4 & 8.9 & 2.6 & 0.162 & 0.87 & 2481 & 15 & +15 \\
\hline
\end{tabular}


Lane et al. Supplementary Papers

Australian Journal of Earth Sciences (2015) 62, 55-75, http://dx.doi.org/10.1080/08120099.2015.993160

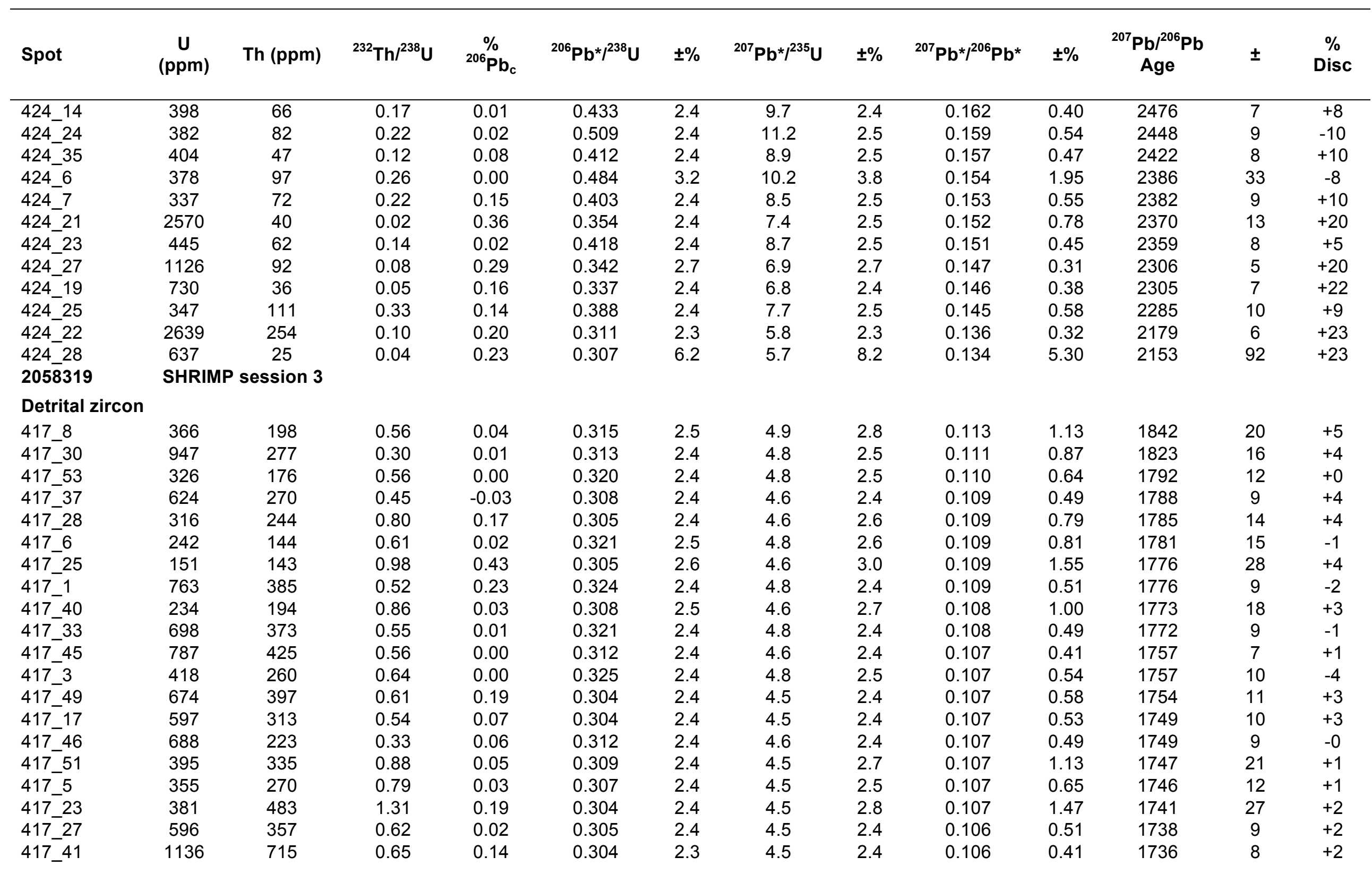


Lane et al. Supplementary Papers

Australian Journal of Earth Sciences (2015) 62, 55-75, http://dx.doi.org/10.1080/08120099.2015.993160

\begin{tabular}{|c|c|c|c|c|c|c|c|c|c|c|c|c|c|}
\hline Spot & $\underset{(p p m)}{U}$ & Th (ppm) & ${ }^{232} \mathrm{Th} /{ }^{238} \mathrm{U}$ & $\begin{array}{c}\% \\
{ }^{206} \mathrm{~Pb}_{\mathrm{c}}\end{array}$ & ${ }^{206} \mathrm{~Pb}{ }^{\star} /{ }^{238} \mathrm{U}$ & $\pm \%$ & $\left.{ }^{207} \mathrm{~Pb}{ }^{\star}\right|^{235} \mathrm{U}$ & $\pm \%$ & $\left.{ }^{207} \mathrm{~Pb}^{\star}\right|^{206} \mathrm{~Pb}^{*}$ & $\pm \%$ & $\begin{array}{c}{ }^{207} \mathrm{~Pb} /{ }^{206} \mathrm{~Pb} \\
\mathrm{Age}\end{array}$ & \pm & $\begin{array}{c}\% \\
\text { Disc }\end{array}$ \\
\hline 417_32 & 298 & 216 & 0.75 & -0.02 & 0.316 & 2.9 & 4.6 & 3.2 & 0.106 & 1.37 & 1736 & 25 & -2 \\
\hline 417_35 & 87 & 39 & 0.46 & -0.11 & 0.310 & 2.7 & 4.5 & 3.1 & 0.106 & 1.37 & 1730 & 25 & -1 \\
\hline 417_13 & 220 & 170 & 0.80 & 0.20 & 0.313 & 2.5 & 4.6 & 2.7 & 0.106 & 0.91 & 1729 & 17 & -2 \\
\hline 417_43 & 368 & 126 & 0.35 & 0.11 & 0.300 & 2.4 & 4.4 & 2.5 & 0.106 & 0.68 & 1727 & 13 & +2 \\
\hline 417_48 & 684 & 449 & 0.68 & 0.20 & 0.298 & 2.4 & 4.3 & 2.4 & 0.105 & 0.52 & 1720 & 10 & +3 \\
\hline 417_2 & 276 & 77 & 0.29 & 0.12 & 0.306 & 2.7 & 4.4 & 2.8 & 0.105 & 0.81 & 1713 & 15 & -1 \\
\hline 417_44 & 371 & 226 & 0.63 & 0.21 & 0.293 & 2.4 & 4.2 & 2.5 & 0.103 & 0.79 & 1685 & 15 & +2 \\
\hline 417_21 & 555 & 556 & 1.04 & 0.29 & 0.286 & 3.0 & 4.0 & 3.1 & 0.101 & 0.71 & 1640 & 13 & +1 \\
\hline \multicolumn{14}{|c|}{ Discordant cores/high Pbc } \\
\hline 417_34 & 600 & 334 & 0.57 & 0.00 & 0.405 & 2.4 & 8.4 & 2.4 & 0.151 & 0.35 & 2356 & 6 & +8 \\
\hline 417_11 & 637 & 206 & 0.33 & 0.19 & 0.370 & 2.7 & 6.8 & 2.8 & 0.133 & 0.48 & 2140 & 8 & +6 \\
\hline 417_47 & 1167 & 132 & 0.12 & 0.93 & 0.317 & 2.7 & 5.1 & 2.8 & 0.116 & 0.92 & 1897 & 17 & +7 \\
\hline 417_22 & 165 & 122 & 0.77 & -0.12 & 0.302 & 2.5 & 4.6 & 2.7 & 0.110 & 1.01 & 1796 & 18 & +6 \\
\hline 417_20 & 766 & 326 & 0.44 & -0.01 & 0.300 & 2.8 & 4.5 & 2.8 & 0.110 & 0.43 & 1792 & 8 & +6 \\
\hline 417_16 & 313 & 56 & 0.18 & 0.22 & 0.301 & 2.4 & 4.5 & 2.6 & 0.109 & 0.84 & 1778 & 15 & +5 \\
\hline 417_7 & 882 & 653 & 0.76 & 0.01 & 0.286 & 2.3 & 4.3 & 2.4 & 0.109 & 0.41 & 1775 & 8 & +10 \\
\hline 417_12 & 670 & 577 & 0.89 & 0.10 & 0.336 & 2.9 & 5.0 & 3.0 & 0.108 & 0.66 & 1774 & 12 & -6 \\
\hline 417_31 & 1284 & 1507 & 1.21 & 0.03 & 0.290 & 2.4 & 4.3 & 2.4 & 0.108 & 0.33 & 1770 & 6 & +8 \\
\hline 417_4 & 603 & 569 & 0.97 & 0.40 & 0.296 & 2.4 & 4.4 & 2.5 & 0.107 & 0.73 & 1757 & 13 & +5 \\
\hline 417_24 & 1208 & 1048 & 0.90 & 0.10 & 0.295 & 2.4 & 4.4 & 2.4 & 0.107 & 0.61 & 1754 & 11 & +6 \\
\hline 417_36 & 687 & 245 & 0.37 & 0.02 & 0.291 & 2.4 & 4.3 & 2.4 & 0.107 & 0.50 & 1753 & 9 & +7 \\
\hline 417_29 & 236 & 162 & 0.71 & -0.07 & 0.340 & 2.5 & 5.0 & 2.7 & 0.107 & 1.03 & 1749 & 19 & -9 \\
\hline 417_42 & 532 & 315 & 0.61 & 0.04 & 0.295 & 2.4 & 4.3 & 2.5 & 0.107 & 0.64 & 1748 & 12 & +5 \\
\hline 417_52 & 520 & 205 & 0.41 & 0.05 & 0.294 & 2.4 & 4.3 & 2.5 & 0.107 & 0.54 & 1743 & 10 & +5 \\
\hline 417_14 & 955 & 919 & 0.99 & 0.55 & 0.314 & 2.4 & 4.6 & 2.6 & 0.106 & 0.99 & 1735 & 18 & -2 \\
\hline 417_50 & 453 & 146 & 0.33 & 0.66 & 0.266 & 2.4 & 3.7 & 2.6 & 0.100 & 0.93 & 1625 & 17 & +7 \\
\hline 417_15 & 835 & 38 & 0.05 & 0.75 & 0.291 & 2.5 & 3.9 & 2.6 & 0.098 & 0.73 & 1581 & 14 & -5 \\
\hline 417_39 & 523 & 144 & 0.28 & 0.69 & 0.215 & 2.4 & 2.9 & 2.7 & 0.098 & 1.25 & 1579 & 23 & +22 \\
\hline \multicolumn{14}{|c|}{ Metamorphic zircon } \\
\hline 417_38 & 128 & 18 & 0.14 & -0.04 & 0.299 & 2.6 & 4.4 & 2.8 & 0.106 & 1.03 & 1740 & 19 & +3 \\
\hline 417_19 & 236 & 26 & 0.12 & 0.00 & 0.294 & 2.5 & 4.3 & 2.6 & 0.106 & 0.78 & 1726 & 14 & +4 \\
\hline 417_18 & 388 & 26 & 0.07 & 0.00 & 0.316 & 2.5 & 4.6 & 2.7 & 0.105 & 0.95 & 1708 & 17 & -4 \\
\hline 417_9 & 323 & 22 & 0.07 & -0.01 & 0.303 & 2.4 & 4.4 & 2.5 & 0.106 & 0.64 & 1726 & 12 & +1 \\
\hline
\end{tabular}




\begin{tabular}{|c|c|c|c|c|c|c|c|c|c|c|c|c|c|}
\hline Spot & $\underset{(p p m)}{U}$ & Th (ppm) & ${ }^{232} \mathrm{Th} /{ }^{238} \mathrm{U}$ & $\begin{array}{c}\% \\
{ }^{206} \mathrm{~Pb}_{\mathrm{c}}\end{array}$ & ${ }^{206} \mathrm{~Pb}^{\star} /{ }^{238} \mathrm{U}$ & $\pm \%$ & $\left.{ }^{207} \mathrm{~Pb}^{\star}\right|^{235} \mathrm{U}$ & $\pm \%$ & $\left.{ }^{207} \mathrm{~Pb}^{\star}\right|^{206} \mathrm{~Pb}^{\star}$ & $\pm \%$ & $\begin{array}{c}{ }^{207} \mathrm{~Pb} /{ }^{206} \mathrm{~Pb} \\
\mathrm{Age}\end{array}$ & \pm & $\begin{array}{c}\% \\
\text { Disc }\end{array}$ \\
\hline \multicolumn{14}{|c|}{ Metamorphic rims } \\
\hline 417_10 & 779 & 151 & 0.20 & 0.05 & 0.316 & 2.6 & 4.6 & 2.7 & 0.107 & 0.43 & 1740 & 8 & -2 \\
\hline 417_26 & 92 & 13 & 0.15 & 0.15 & 0.305 & 2.7 & 4.5 & 3.0 & 0.106 & 1.40 & 1740 & 26 & +2 \\
\hline $183 \overline{9} 526$ & \multicolumn{13}{|c|}{ SHRIMP session 4} \\
\hline \multicolumn{14}{|c|}{ Detrital zircon } \\
\hline 526.20 .1 .1 & 245 & 108 & 0.46 & 0.02 & 0.474 & 1.1 & 10.81 & 1.1 & 0.1653 & 0.28 & 2510 & 5 & +0 \\
\hline 526.18 .1 .1 & 315 & 173 & 0.57 & 0.00 & 0.430 & 1.1 & 8.69 & 1.1 & 0.1467 & 0.27 & 2307 & 5 & +0 \\
\hline 526.1 .1 .1 & 107 & 73 & 0.70 & 0.13 & 0.353 & 1.2 & 5.92 & 1.4 & 0.1218 & 0.59 & 1983 & 10 & +2 \\
\hline 526.11 .1 .1 & 323 & 90 & 0.29 & 0.00 & 0.357 & 1.1 & 5.97 & 1.1 & 0.1212 & 0.31 & 1974 & 6 & +0 \\
\hline 526.12 .1 .1 & 249 & 99 & 0.41 & 0.07 & 0.329 & 1.9 & 5.17 & 2.0 & 0.1142 & 0.40 & 1868 & 7 & +2 \\
\hline 526.9.1.1 & 237 & 80 & 0.35 & 0.23 & 0.314 & 1.1 & 4.91 & 1.2 & 0.1134 & 0.49 & 1854 & 9 & +6 \\
\hline 526.30 .1 .1 & 438 & 167 & 0.39 & 0.01 & 0.324 & 2.8 & 5.03 & 2.8 & 0.1124 & 0.46 & 1838 & 8 & +2 \\
\hline 526.5 .1 .1 & 179 & 79 & 0.46 & 0.03 & 0.317 & 1.2 & 4.89 & 1.2 & 0.1118 & 0.47 & 1829 & 9 & +3 \\
\hline 526.10 .2 .1 & 268 & 108 & 0.42 & 0.03 & 0.328 & 1.1 & 5.03 & 1.2 & 0.1113 & 0.40 & 1821 & 7 & -0 \\
\hline 526.44 .1 .1 & 257 & 103 & 0.42 & 0.01 & 0.321 & 1.1 & 4.92 & 1.2 & 0.1113 & 0.37 & 1820 & 7 & +2 \\
\hline 526.42 .1 .1 & 202 & 61 & 0.31 & 0.21 & 0.323 & 1.1 & 4.93 & 1.2 & 0.1108 & 0.52 & 1812 & 10 & +0 \\
\hline 526.41 .1 .1 & 212 & 87 & 0.42 & -0.01 & 0.314 & 1.1 & 4.77 & 1.2 & 0.1101 & 0.42 & 1801 & 8 & +3 \\
\hline 526.31 .1 .1 & 153 & 72 & 0.48 & 0.19 & 0.308 & 1.2 & 4.67 & 1.3 & 0.1100 & 0.64 & 1800 & 12 & +4 \\
\hline 526.23 .1 .1 & 293 & 85 & 0.30 & 0.17 & 0.317 & 1.1 & 4.80 & 1.2 & 0.1099 & 0.44 & 1798 & 8 & +1 \\
\hline 526.40 .1 .1 & 202 & 98 & 0.50 & 0.06 & 0.316 & 1.1 & 4.78 & 1.2 & 0.1097 & 0.44 & 1795 & 8 & +2 \\
\hline 526.3.1.1 & 165 & 45 & 0.28 & 0.02 & 0.314 & 1.2 & 4.75 & 1.5 & 0.1095 & 0.94 & 1791 & 17 & +2 \\
\hline 526.27 .1 .1 & 170 & 64 & 0.39 & -0.02 & 0.308 & 1.2 & 4.62 & 1.3 & 0.1089 & 0.50 & 1781 & 9 & +3 \\
\hline 526.6.1.1 & 118 & 56 & 0.49 & 0.09 & 0.315 & 1.2 & 4.73 & 1.6 & 0.1088 & 1.07 & 1779 & 19 & +1 \\
\hline 526.46 .1 .1 & 281 & 66 & 0.24 & 0.04 & 0.315 & 1.1 & 4.72 & 1.2 & 0.1086 & 0.38 & 1777 & 7 & +1 \\
\hline 526.4.1.1 & 155 & 91 & 0.60 & 0.05 & 0.314 & 1.2 & 4.70 & 1.5 & 0.1086 & 0.93 & 1776 & 17 & +1 \\
\hline 526.60 .1 .1 & 262 & 113 & 0.45 & 0.22 & 0.308 & 1.1 & 4.61 & 1.6 & 0.1086 & 1.21 & 1776 & 22 & +3 \\
\hline 526.38 .1 .1 & 198 & 184 & 0.96 & 0.24 & 0.290 & 1.1 & 4.33 & 1.3 & 0.1082 & 0.56 & 1770 & 10 & +8 \\
\hline 526.24 .1 .1 & 238 & 152 & 0.66 & 0.01 & 0.309 & 1.1 & 4.61 & 1.2 & 0.1081 & 0.42 & 1768 & 8 & +2 \\
\hline 526.13 .1 .1 & 101 & 92 & 0.94 & 0.07 & 0.303 & 1.2 & 4.51 & 1.4 & 0.1081 & 0.66 & 1767 & 12 & +4 \\
\hline
\end{tabular}




\begin{tabular}{|c|c|c|c|c|c|c|c|c|c|c|c|c|c|}
\hline Spot & $\underset{(p p m)}{U}$ & Th (ppm) & ${ }^{232} \mathrm{Th} /{ }^{238} \mathrm{U}$ & $\begin{array}{c}{ }^{\%} \% \\
{ }^{206} \mathrm{~Pb}_{\mathrm{c}}\end{array}$ & ${ }^{206} \mathrm{~Pb}^{\star} I^{238} \mathrm{U}$ & $\pm \%$ & $\left.{ }^{207} \mathrm{~Pb}^{*}\right|^{235} \mathrm{U}$ & $\pm \%$ & $\left.{ }^{207} \mathrm{~Pb}^{\star}\right|^{206} \mathrm{~Pb}^{*}$ & $\pm \%$ & $\begin{array}{c}{ }^{207} \mathrm{~Pb} /{ }^{206} \mathrm{~Pb} \\
\mathrm{Age}\end{array}$ & \pm & $\begin{array}{c}\% \\
\text { Disc }\end{array}$ \\
\hline 526.37 .1 .1 & 386 & 333 & 0.89 & 0.26 & 0.303 & 1.1 & 4.51 & 1.2 & 0.1079 & 0.54 & 1764 & 10 & +4 \\
\hline 526.21 .1 .1 & 243 & 226 & 0.96 & 0.00 & 0.308 & 1.1 & 4.58 & 1.2 & 0.1077 & 0.40 & 1761 & 7 & +2 \\
\hline 526.59 .1 .1 & 319 & 91 & 0.29 & 0.41 & 0.296 & 1.1 & 4.39 & 1.2 & 0.1076 & 0.48 & 1760 & 9 & +6 \\
\hline 526.53 .1 .1 & 144 & 385 & 2.76 & 0.14 & 0.308 & 1.2 & 4.57 & 1.3 & 0.1075 & 0.59 & 1758 & 11 & +2 \\
\hline 526.50 .1 .1 & 196 & 92 & 0.49 & 0.00 & 0.307 & 1.1 & 4.55 & 1.2 & 0.1075 & 0.44 & 1757 & 8 & +2 \\
\hline 526.35 .1 .1 & 162 & 143 & 0.91 & 0.03 & 0.313 & 1.2 & 4.62 & 1.3 & 0.1072 & 0.50 & 1753 & 9 & -0 \\
\hline 526.45 .1 .1 & 120 & 107 & 0.92 & -0.02 & 0.316 & 1.2 & 4.66 & 1.3 & 0.1070 & 0.57 & 1749 & 10 & -1 \\
\hline 526.48 .1 .1 & 375 & 70 & 0.19 & 0.11 & 0.302 & 1.1 & 4.46 & 1.1 & 0.1069 & 0.38 & 1748 & 7 & +3 \\
\hline 526.29 .1 .1 & 186 & 183 & 1.02 & 0.04 & 0.310 & 1.1 & 4.57 & 1.2 & 0.1067 & 0.47 & 1744 & 9 & +0 \\
\hline 526.39 .1 .1 & 185 & 202 & 1.13 & 0.05 & 0.304 & 1.1 & 4.47 & 1.2 & 0.1067 & 0.48 & 1743 & 9 & +2 \\
\hline 526.33 .1 .1 & 130 & 70 & 0.55 & 0.03 & 0.311 & 1.2 & 4.57 & 1.3 & 0.1066 & 0.56 & 1742 & 10 & -0 \\
\hline 526.19.1.1 & 141 & 107 & 0.78 & 0.01 & 0.305 & 1.2 & 4.49 & 1.3 & 0.1066 & 0.54 & 1741 & 10 & +2 \\
\hline 526.22 .1 .1 & 102 & 113 & 1.15 & 0.08 & 0.310 & 1.2 & 4.55 & 1.4 & 0.1065 & 0.67 & 1741 & 12 & +0 \\
\hline 526.26.1.1 & 202 & 134 & 0.68 & 0.10 & 0.307 & 1.1 & 4.51 & 1.2 & 0.1065 & 0.47 & 1740 & 9 & +1 \\
\hline 526.7.1.1 & 223 & 49 & 0.23 & 0.00 & 0.309 & 1.1 & 4.53 & 1.2 & 0.1063 & 0.42 & 1738 & 8 & +0 \\
\hline 526.14 .1 .1 & 249 & 145 & 0.60 & 0.50 & 0.310 & 1.1 & 4.55 & 1.3 & 0.1062 & 0.58 & 1736 & 11 & -0 \\
\hline 526.2 .1 .1 & 120 & 78 & 0.67 & 0.08 & 0.306 & 1.2 & 4.49 & 1.4 & 0.1062 & 0.63 & 1735 & 12 & +1 \\
\hline 526.49 .1 .1 & 257 & 239 & 0.96 & 0.15 & 0.296 & 1.1 & 4.34 & 1.2 & 0.1062 & 0.56 & 1735 & 10 & +4 \\
\hline \multicolumn{14}{|c|}{ Discordant zircon/high Pbc } \\
\hline 526.56 .1 .1 & 49 & 70 & 1.49 & 57.77 & 4.288 & 44.5 & 260.74 & 54.5 & 0.4410 & 31 & 4055 & 468 & -390 \\
\hline 526.47 .1 .1 & 422 & 334 & 0.82 & 5.39 & 0.271 & 1.4 & 4.26 & 2.5 & 0.1141 & 2.05 & 1865 & 37 & +19 \\
\hline 526.10 .1 .1 & 500 & 162 & 0.34 & 1.30 & 0.254 & 1.3 & 3.93 & 1.4 & 0.1122 & 0.61 & 1836 & 11 & +23 \\
\hline 526.32 .1 .1 & 460 & 167 & 0.38 & 7.16 & 0.222 & 2.2 & 3.44 & 4.7 & 0.1121 & 4.22 & 1834 & 77 & +32 \\
\hline 526.52 .1 .1 & 421 & 465 & 1.14 & 5.52 & 0.230 & 4.6 & 3.45 & 5.4 & 0.1090 & 2.86 & 1783 & 52 & +28 \\
\hline 526.15 .1 .1 & 348 & 220 & 0.65 & 0.84 & 0.293 & 1.6 & 4.39 & 2.0 & 0.1089 & 1.16 & 1781 & 21 & +8 \\
\hline 526.16 .1 .1 & 359 & 564 & 1.63 & 8.41 & 0.222 & 1.1 & 3.32 & 4.4 & 0.1087 & 4.28 & 1777 & 78 & +30 \\
\hline 526.58 .1 .1 & 473 & 628 & 1.37 & 7.25 & 0.241 & 1.9 & 3.61 & 2.3 & 0.1084 & 1.30 & 1772 & 24 & +24 \\
\hline 526.34 .1 .1 & 373 & 358 & 0.99 & 2.79 & 0.260 & 1.1 & 3.88 & 4.4 & 0.1083 & 4.30 & 1771 & 78 & +18 \\
\hline 526.8 .2 .1 & 591 & 240 & 0.42 & 4.48 & 0.217 & 1.1 & 3.23 & 1.9 & 0.1081 & 1.61 & 1767 & 29 & +31 \\
\hline
\end{tabular}


Lane et al. Supplementary Papers

Australian Journal of Earth Sciences (2015) 62, 55-75, http://dx.doi.org/10.1080/08120099.2015.993160

\begin{tabular}{|c|c|c|c|c|c|c|c|c|c|c|c|c|c|}
\hline Spot & $\underset{(p p m)}{U}$ & Th (ppm) & ${ }^{232} \mathrm{Th} /{ }^{238} \mathrm{U}$ & $\begin{array}{c}\% \\
{ }^{206} \mathrm{~Pb}_{\mathrm{c}}\end{array}$ & ${ }^{206} \mathrm{~Pb}^{\star} I^{238} \mathrm{U}$ & $\pm \%$ & ${ }^{207} \mathrm{~Pb}^{\star} I^{235} \mathrm{U}$ & $\pm \%$ & $\left.{ }^{207} \mathrm{~Pb}^{\star}\right|^{206} \mathrm{~Pb}^{*}$ & $\pm \%$ & $\begin{array}{c}{ }^{207} \mathrm{~Pb} /{ }^{206} \mathrm{~Pb} \\
\text { Age }\end{array}$ & \pm & $\begin{array}{c}\% \\
\text { Disc }\end{array}$ \\
\hline 526.25 .1 .1 & 432 & 314 & 0.75 & 1.68 & 0.253 & 2.0 & 3.76 & 2.5 & 0.1080 & 1.59 & 1767 & 29 & +20 \\
\hline 526.57 .1 .1 & 362 & 341 & 0.97 & 3.18 & 0.262 & 1.1 & 3.90 & 2.6 & 0.1079 & 2.40 & 1765 & 44 & +17 \\
\hline 526.62 .1 .1 & 411 & 265 & 0.67 & 4.31 & 0.275 & 1.8 & 4.09 & 2.1 & 0.1078 & 0.99 & 1763 & 18 & +12 \\
\hline 526.17 .1 .1 & 283 & 184 & 0.67 & 1.88 & 0.302 & 1.1 & 4.46 & 1.7 & 0.1071 & 1.24 & 1751 & 23 & +3 \\
\hline 526.43 .1 .1 & 314 & 155 & 0.51 & 0.57 & 0.298 & 1.1 & 4.39 & 1.2 & 0.1070 & 0.52 & 1748 & 10 & +4 \\
\hline 526.8 .1 .1 & 306 & 47 & 0.16 & 0.73 & 0.274 & 1.1 & 4.04 & 1.6 & 0.1069 & 1.20 & 1748 & 22 & +12 \\
\hline 526.54 .1 .1 & 317 & 207 & 0.67 & 0.84 & 0.304 & 1.1 & 4.46 & 1.2 & 0.1063 & 0.56 & 1737 & 10 & +2 \\
\hline 526.28 .1 .1 & 385 & 252 & 0.68 & 0.74 & 0.300 & 1.1 & 4.39 & 1.2 & 0.1063 & 0.54 & 1737 & 10 & +3 \\
\hline 526.51 .1 .1 & 395 & 354 & 0.93 & 2.98 & 0.236 & 1.9 & 3.46 & 2.9 & 0.1063 & 2.19 & 1737 & 40 & +24 \\
\hline 526.55 .1 .1 & 319 & 306 & 0.99 & 3.46 & 0.269 & 1.1 & 3.94 & 1.8 & 0.1061 & 1.36 & 1734 & 25 & +13 \\
\hline 526.61 .1 .1 & 284 & 357 & 1.30 & 6.53 & 0.291 & 1.5 & 4.22 & 5.0 & 0.1051 & 4.72 & 1716 & 87 & +4 \\
\hline
\end{tabular}


Table $2 \mathrm{Sm}-\mathrm{Nd}$ isotope data.

\begin{tabular}{|c|c|c|c|c|c|c|c|}
\hline Sample Number & 2058318 & 2058319 & 2058328 & 2017472 & 1839526 & 2017372 & 2017473 \\
\hline Input age of rock T (Ma) & 1750 & 1750 & 1750 & 1750 & 1750 & 1750 & 1750 \\
\hline Unmixed ${ }^{143} \mathrm{Nd} /{ }^{144} \mathrm{Nd}$ & 0.511304 & 0.511410 & 0.511440 & 0.511489 & 0.511450 & 0.511445 & 0.511431 \\
\hline Nd ugg-1 & 46.3 & 37.8 & 29.1 & 36.6 & 41.9 & 26.6 & 57.5 \\
\hline Sm ugg-1 & 7.8 & 6.7 & 5.2 & 6.8 & 7.4 & 4.9 & 10.0 \\
\hline${ }^{147} \mathrm{Sm} /{ }^{144} \mathrm{Nd}$ & 0.1013 & 0.1078 & 0.1073 & 0.1119 & 0.1066 & 0.1117 & 0.1049 \\
\hline eNd $(T=0)$ & -26.03 & -23.95 & -23.37 & -22.41 & -23.18 & -23.27 & -23.54 \\
\hline${ }^{143} \mathrm{Nd} /{ }^{144} \mathrm{Nd}(\mathrm{T})$ & 0.510137 & 0.510169 & 0.510205 & 0.510201 & 0.510222 & 0.510160 & 0.510224 \\
\hline eNd $(T)$ & -4.66 & -4.04 & -3.33 & -3.41 & -3.00 & -4.22 & -2.97 \\
\hline TDM (Ma) & 2475 & 2474 & 2419 & 2456 & 2392 & 2514 & 2380 \\
\hline TCHUR (Ma) & 2127 & 2101 & 2037 & 2061 & 2007 & 2133 & 2000 \\
\hline $\mathrm{DM}$ at age of rock $(\mathrm{T})$ & 0.510681 & 0.510681 & 0.510681 & 0.510681 & 0.510681 & 0.510681 & 0.510681 \\
\hline CHUR at age of rock $(\mathrm{T})$ & 0.510375 & 0.510375 & 0.510375 & 0.510375 & 0.510375 & 0.510375 & 0.510375 \\
\hline \multicolumn{5}{|c|}{ JNdi-1 reference material measured $28 / 10 / 2013,{ }^{143} \mathrm{Nd} /{ }^{144} \mathrm{Nd}=0.512098$} & \multicolumn{3}{|c|}{9 (2se), 1 measurement. } \\
\hline Sm Nd spike & F QCD & F QCD & F QCD & F QCD & F QCD & F QCD & F QCD \\
\hline Sample wt (g) & 0.12967 & 0.10352 & 0.13699 & 0.10371 & 0.15277 & 0.11300 & 0.13067 \\
\hline Sm Nd spike wt (g) & 0.53987 & 0.57624 & 0.52791 & 0.52592 & 0.52295 & 0.54293 & 0.48945 \\
\hline Measured ${ }^{143} \mathrm{Nd} /{ }^{144} \mathrm{Nd}$ & 0.511327 & 0.511449 & 0.511475 & 0.511526 & 0.511471 & 0.511493 & 0.511449 \\
\hline $2 \mathrm{se}\left(\mathrm{x}^{-6} \mathrm{E}^{-6}\right)$ & 9 & 8 & 8 & 9 & 7 & 9 & 8 \\
\hline Measured ${ }^{150} \mathrm{Nd} /{ }^{144} \mathrm{Nd}$ & 0.341514 & 0.408141 & 0.390751 & 0.398192 & 0.331893 & 0.447456 & 0.312516 \\
\hline 2 se $\left(x 1 E^{-6}\right)$ & 86 & 24 & 34 & 48 & 23 & 27 & 39 \\
\hline Measured ${ }^{147} \mathrm{Sm} /{ }^{149} \mathrm{Sm}$ & 3.22746 & 4.35147 & 4.03903 & 4.05709 & 2.94106 & 4.94970 & 2.59004 \\
\hline 2 se $\left(x 1 E^{-6}\right)$ & 43 & 76 & 64 & 53 & 15 & 56 & 30 \\
\hline Measured ${ }^{152} \mathrm{Sm} /{ }^{149} \mathrm{Sm}$ & 1.90118 & 1.89574 & 1.89906 & 1.89607 & 1.90039 & 1.89148 & 1.90511 \\
\hline 2 se $\left(x 1 E^{-6}\right)$ & 40 & 64 & 27 & 8 & 18 & 48 & 46 \\
\hline
\end{tabular}

